\title{
A Fresh Perspective on Canonical Extensions for Bounded Lattices
}

\section{A. P. K. Craig, M. Haviar \& H. A. Priestley}

\section{Applied Categorical Structures}

A Journal Devoted to Applications of Categorical Methods in Algebra, Analysis, Order, Topology and Computer Science

ISSN 0927-2852

Volume 21

Number 6

Appl Categor Struct (2013) 21:725-749

DOI 10.1007/s10485-012-9287-2
ISSN 0927-2852

\section{APPLIED \\ CATEGORICAL STRUCTURES}

A Journal Devoted to Applications of Categorical Methods in Algebra, Analysis, Order, Topology and Computer Science

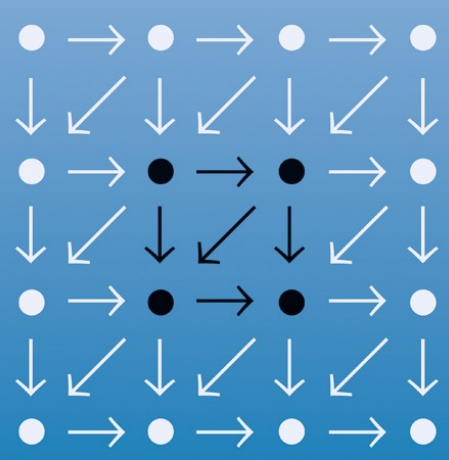

Volume 21, No. 6, December 2013

Springer 
Your article is protected by copyright and all rights are held exclusively by Springer Science+Business Media B.V.. This e-offprint is for personal use only and shall not be selfarchived in electronic repositories. If you wish to self-archive your article, please use the accepted manuscript version for posting on your own website. You may further deposit the accepted manuscript version in any repository, provided it is only made publicly available 12 months after official publication or later and provided acknowledgement is given to the original source of publication and a link is inserted to the published article on Springer's website. The link must be accompanied by the following text: "The final publication is available at link.springer.com". 


\title{
A Fresh Perspective on Canonical Extensions for Bounded Lattices
}

\author{
A. P. K. Craig • M. Haviar • H. A. Priestley
}

Received: 26 September 2011 / Accepted: 30 April 2012 / Published online: 26 May 2012

(C) Springer Science+Business Media B.V. 2012

\begin{abstract}
This paper presents a novel treatment of the canonical extension of a bounded lattice, in the spirit of the theory of natural dualities. At the level of objects, this can be achieved by exploiting the topological representation due to M. Ploščica, and the canonical extension can be obtained in the same manner as can be done in the distributive case by exploiting Priestley duality. To encompass both objects and morphisms the Ploščica representation is replaced by a duality due to Allwein and Hartonas, recast in the style of Ploščica's paper. This leads to a construction of canonical extension valid for all bounded lattices, which is shown to be functorial, with the property that the canonical extension functor decomposes as the composite of two functors, each of which acts on morphisms by composition, in the manner of hom-functors.
\end{abstract}

Keywords Canonical extension $\cdot$ Natural duality $\cdot$ Topological representation

Mathematics Subject Classifications (2010) $08 \mathrm{C} 20 \cdot 06 \mathrm{~B} 23 \cdot 06 \mathrm{D} 50$

The first author gratefully acknowledges funding from the Rhodes Trust. The second author acknowledges support from Slovak grants VEGA 1/0485/09 and APVV-0223-10.

A. P. K. Craig $(\varangle) \cdot$ H. A. Priestley

Mathematical Institute, University of Oxford, 24/29 St. Giles, Oxford OX1 3LB, UK

e-mail: craig@maths.ox.ac.uk

H. A. Priestley

e-mail: hap@maths.ox.ac.uk

M. Haviar

Faculty of Natural Sciences, Matej Bel University, Tajovského 40,

97401 Banská Bystrica, Slovak Republic

e-mail: miroslav.haviar@umb.sk 


\section{Introduction}

Two valuable tools have been developed to study lattice-based algebras: the theory of canonical extensions and the theory of topological dualities. Although the two methodologies have largely been developed separately, they have a symbiotic relationship. In this paper we present a new approach to the canonical extension of a bounded lattice, based on the dual representation due to Ploščica [12]. This yields a construction with strong affinities with the theory of natural dualities-one which has clear categorical merits.

We shall consider only lattices which are bounded, and include the universal bounds, 0 and 1 , in the signature; we denote the variety of all such lattices by $\mathcal{L}$. The subvariety of $\mathcal{L}$ consisting of distributive lattices is denoted by $\mathcal{D}$. The canonical extension of a member $\mathbf{L}$ of $\mathcal{L}$ is a particular lattice completion $\mathbf{L}^{\delta}$ of $\mathbf{L}$, having two properties, known as density and compactness; we recall the definitions below. These properties characterise this completion uniquely, up to an isomorphism fixing the original lattice.

The existence of canonical extensions for members of $\mathcal{L}$ was first established by Gehrke and Harding [7], drawing on the theory of Galois connections; specifically, $\mathbf{L}^{\delta}$ arises as the complete lattice of Galois-stable sets associated with the polarity $R$ between the filter lattice $\operatorname{Filt}(\mathbf{L})$ and the ideal lattice $\operatorname{Idl}(\mathbf{L})$ of $\mathbf{L}$ given by $(F, I) \in R$ if and only if $F \cap I \neq \emptyset$. Further insight into the relationship between $\mathbf{L}, \operatorname{Filt}(\mathbf{L}), \operatorname{Idl}(\mathbf{L})$ and $\mathbf{L}^{\delta}$ was supplied by Gehrke and Priestley [9].

The original investigation by Gehrke and Jónsson [8] of canonical extensions in the distributive case built $\mathbf{L}^{\delta}$ by exploiting Priestley duality. Of course, uniqueness of the canonical extension ensures that this construction leads to the same completion as do the (specialisations of) the constructions in [7] and [9]. However the translations between the various concrete realisations of the canonical extension of a distributive lattice are rather indirect. More significantly, there are obstacles to extending, in a fully satisfactory and transparent way, the duality approach beyond the distributive case. Therefore we contend that it is worthwhile to explore in greater detail than hitherto the interface between canonical extensions and duality theory for lattices, and a categorical context for these.

Each of Priestley duality for $\mathcal{D}$ and Stone duality for Boolean algebras, $\mathcal{B}$, is an instance of a natural duality. Indeed, these dualities provided prototypical examples for the general theory, as presented in the text of Clark and Davey [3]. In its basic form, the theory of natural dualities applies to a prevariety $\mathcal{A}=\mathbb{I S P}(\mathbf{M})$, where $\mathbf{M}$ is some finite algebra. One sets up functors $\mathrm{D}: \mathcal{A} \rightarrow \boldsymbol{X}_{\mathcal{T}}$ and $\mathrm{E}: \boldsymbol{X}_{\mathcal{T}} \rightarrow \mathcal{A}$ between $\mathcal{A}$ and a suitable category $\boldsymbol{X}_{\mathcal{T}}$ of structured Boolean spaces. Here D and E are homfunctors into, respectively, $\mathbf{M}$ and $\underset{\sim}{\mathbf{M}_{\mathcal{T}}}$, where $\mathbf{\sim}_{\mathcal{T}}$ is an alter ego for $\mathbf{M}$, that is, an object in $\boldsymbol{X}_{\mathcal{T}}$ whose underlying set is the same as that of $\mathbf{M}$. If, for a suitable choice of $\mathbf{M}_{\mathcal{T}}$, we obtain $\mathbf{A} \cong \operatorname{ED}(\mathbf{A})$ for all $\mathbf{A} \in \mathcal{A}$, then we say we have a duality; this suffices to give a topological representation for the members of $\mathcal{A}$. (Optimally, one also has $\mathbf{X} \cong \mathrm{DE}(\mathbf{X})$ for all $\mathbf{X} \in \mathcal{X}_{\mathcal{T}}$ and hence a dual category equivalence between $\mathcal{A}$ and $\boldsymbol{X}_{\mathcal{T}}$, but such full dualities are not of relevance here.) In the case of $\mathcal{B}$ and $\mathcal{D}$, the algebra $\mathbf{M}$ is a two-element algebra, and ${\underset{\sim}{\mathcal{T}}}_{\mathcal{T}}$ is the set $\{0,1\}$ equipped with the discrete topology and, respectively, no relations and a single relation, viz. the order relation $\leqslant$ with $0<1$. The functors $\mathrm{D}$ and $\mathrm{E}$ act on morphisms by composition, and this is a key feature of the way in which the duality operates. Moreover, by modifying 
the functors $\mathrm{D}$ and $\mathrm{E}$, one arrives at a functorial construction of the canonical extension-one which makes overt its categorical relationship to the duality. The canonical extension construction can then be viewed in the way shown in Fig. 1. In the figure, $b$ denotes the functor forgetting the topology and the functor $G$ acts in the same manner as E does, but on untopologised rather than topologised structures. The codomain $\mathcal{D}^{+}$of $\mathrm{G}$ can be taken to be the category of doubly algebraic distributive lattices with complete lattice homomorphisms. In fact, more is true: there is a homfunctor $F$ adjoint to $G$ such that $F$ and $G$ set up a dual equivalence between $\mathcal{D}^{+}$ and $\mathcal{X}$ (see $[5,6]$ ). However the functor $\mathrm{F}$ is not involved in the factorisation of $\delta$. On the other hand, we do make use of $\mathrm{E}$ :

$$
\mathbf{L} \cong \mathrm{ED}(\mathbf{L}) \subseteq \mathbf{L}^{\delta} \quad \text { for all } \mathbf{L} \in \mathcal{D} .
$$

Unlike $\mathcal{B}$ and $\mathcal{D}$, the variety $\mathcal{L}$ of bounded lattices is not finitely generated. This prevents it from coming within the scope of the theory of natural dualities. However there is available a long-established representation of bounded lattices due to Urquhart [14]. This does not stem from a dual category equivalence but it does provide a concrete topological representation of each $\mathbf{L} \in \mathcal{L}$. Of greater relevance to us here, though, is the recasting of Urquhart's work by Ploščica [12]. This was motivated by natural duality theory, and is in the spirit of that theory. In barest outline, Ploščica's extension of the Priestley representation is accomplished by replacing total maps into $\{0,1\}$, qua lattice or qua partially ordered set, by appropriate maximally-defined partial maps of the same sort. One may then conjecture that, mutatis mutandis, the canonical extension of an arbitrary bounded lattice may be obtained in the same manner as in Fig. 1 by 'forgetting the topology' at the level of the Ploščica first dual. The principal result of Section 3, Theorem 3.11, confirms that this is indeed the case. In preparation, Section 2 summarises the key notions presented in Ploščica's paper [12], investigates the basic properties of the corresponding concepts for the topology-free setting, and reveals the interplay between the topologised and untopologised versions. We emphasise that the validation of our construction of the canonical extension is independent of pre-existing theory of canonical extensions for $\mathcal{L}$ : we rely solely on the characteristic density and compactness properties, to ensure that, for $\mathbf{L} \in \mathcal{L}$, our candidate for $\mathbf{L}^{\delta}$ contains an isomorphic copy of $\mathbf{L}$. (A detailed discussion of the role of polarities in our framework, and a reconciliation of alternative approaches will be presented separately (Craig and Haviar [4]).)

So, at the object level, we have a construction which mimics the duality technnique used in the distributive case. But it is with morphisms that obstacles arise with duality theory for $\mathcal{L}$. We are nonetheless able to present a functorial construction, and one in which the canonical extension functor factorises in a manner analogous to that shown

Fig. 1 Factorising the canonical extension functor on $\mathcal{D}$

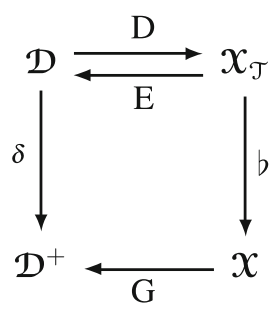


in Fig. 1. Some sacrifice is unavoidable to achieve this, and in our approach this is the enlargement of the first dual space of $\mathbf{L}$. Specifically, our factorisation aligns with the duality for $\mathcal{L}$ due to Allwein and Hartonas [1,2] and so it is expedient to recast the Allwein and Hartonas representation at the object level in the Ploščica style. We retain a critical feature of the natural duality framework, viz. that the functors involved behave very like hom-functors. By saying a functor, $\mathrm{H}$ say, is 'very like' a hom-functor, we mean that there is some fixed target, $\mathrm{N}$ say (not necessarily in the codomain of $\mathrm{H}$ ), such that $\mathrm{H}$ sends an object $C$ to a specified set of partial maps from $C$ into $\mathrm{N}$ and that $\mathrm{H}$ acts on morphisms by composition. Thus we are able to retain key features present in the distributive case at the cost of amending the ancillary functors into which the functor ${ }^{\delta}$ splits, but without having to change the very natural way in which these functors act (see Fig. 3 in Section 4). The major work involved in carrying out our programme is in verifying that the functors we require are well-defined. Once this is done, the lifting of homomorphisms to canonical extensions goes through very cleanly. We could have worked with the Allwein and Hartonas duality from the outset and essentially bypassed Ploščica's representation. We have elected not to do this for two reasons. Firstly, in certain applications morphisms are not important, and it is then advantageous to have a construction which coincides in the distributive case with the usual duality-based one. Secondly, we are able to convert the Ploščica-style construction at the object level to one based on the enlarged first dual space, and so do not need to start afresh to encompass morphisms.

We note that canonical extensions of lattices provide a platform for studying lattice-based algebras (also known as lattice expansions). A valuable recent survey of canonical extensions for lattice-based algebras, including a discussion of their important role in the semantic modelling of logics, is given by Gehrke and Vosmaer [10].

\section{The Framework for the Construction}

In this section we set up the framework within which we shall construct the canonical extension of a bounded lattice $\mathbf{L}$. In outline, we look at the structures, and associated maps, obtained by deleting the topology from the dual spaces employed in Ploščica's representation. We thereby arrive at a complete lattice which we shall later show serves as $\mathbf{L}^{\delta}$.

We begin by recalling some basic definitions concerning completions. A completion of a (bounded) lattice $\mathbf{L}$ is defined to be a pair $(e, C)$ where $C$ is a complete lattice and $e: L \hookrightarrow C$ is an embedding. An element of a completion $(e, C)$ of a (bounded) lattice $L$ which is representable as a meet (join) of elements from $e(L)$ is called a filter element (ideal element). Filter (ideal) elements are called closed (open) elements in the older literature. The sets of such elements will be denoted $\mathbb{F}(C)$ and $\mathbb{I}(C)$, respectively. A completion $(e, C)$ of $L$ is said to be dense if every element of $C$ is both a join of meets and a meet of joins of elements from $e(L)$; it is said to be compact if, for any sets $A, B \subseteq L$ with $\bigwedge e(A) \leqslant \bigvee e(B)$, there exist finite subsets $A^{\prime} \subseteq A$ and $B^{\prime} \subseteq B$ such that $\bigwedge e\left(A^{\prime}\right) \leqslant \bigvee e\left(B^{\prime}\right)$. A canonical extension of $\mathbf{L}$ is a dense and compact completion. As noted above, every bounded lattice $\mathbf{L}$ has a canonical extension and any two canonical extensions of $\mathbf{L}$ are isomorphic via an isomorphism that fixes the elements of $\mathbf{L}$. 
The central idea in Ploščica's representation of bounded lattices [12] is the replacement of total maps by partial maps. Let $\mathbf{L}_{1}, \mathbf{L}_{2} \in \mathcal{L}$. A partial map $f: \mathbf{L}_{1} \rightarrow$ $\mathbf{L}_{2}$ between bounded lattices is called a partial homomorphism if its domain is a 0,1 -sublattice of $\mathbf{L}_{1}$ and $f: \operatorname{dom}(f) \rightarrow \mathbf{L}_{2}$ is an $\mathcal{L}$-homomorphism. A partial homomorphism is said to be maximal if there is no partial homomorphism properly extending it; such a map is referred to as an MPH for short. By Zorn's Lemma, every partial homomorphism can be extended to an MPH. For bounded lattices $\mathbf{L}$ and $\mathbf{K}$, we denote by $\mathcal{L}^{\mathrm{mp}}(\mathbf{L}, \mathbf{K})$ the set of all MPH's from $\mathbf{L}$ to $\mathbf{K}$.

Let

$$
\underline{\mathbf{2}}:=\langle\{0,1\} ; \vee, \wedge, 0,1\rangle \text { and } \underset{\sim}{\mathbf{2}}:=\langle\{0,1\} ; \leqslant\rangle
$$

denote, respectively, the two-element bounded lattice and the two-element ordered

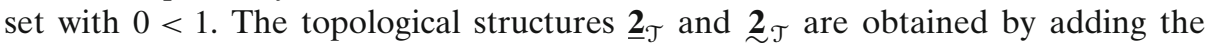
discrete topology $\mathcal{T}$ to $\underline{\mathbf{2}}$ and $\underset{\sim}{\mathbf{2}}$, respectively.

Following Ploščica [12], for any bounded lattice $\mathbf{L}$, the topological dual space of $\mathbf{L}$ is defined in the following way. We equip the set $\mathcal{L}^{\mathrm{mp}}(\mathbf{L}, \underline{\mathbf{2}})$ with the binary relation $E$ defined by the rule

$$
(f, g) \in E \quad \text { iff } f(x) \leqslant g(x) \text { for every } x \in \operatorname{dom}(f) \cap \operatorname{dom}(g) ;
$$

when needed, we also endow it with the topology $\mathcal{T}$ which has a subbasis of closed sets all sets of the form

$$
V_{a}=\left\{f \in \mathcal{L}^{\mathrm{mp}}(\mathbf{L}, \underline{\mathbf{2}}) \mid f(a)=0\right\} \quad \text { and } \quad W_{a}=\left\{f \in \mathcal{L}^{\mathrm{mp}}(\mathbf{L}, \underline{\mathbf{2}}) \mid f(a)=1\right\},
$$

where $a \in L$. We let $\mathrm{D}(\mathbf{L})=\left(\mathcal{L}^{\mathrm{mp}}(\mathbf{L}, \underline{\mathbf{2}}), E, \mathcal{T}\right)$. The topology of $\mathrm{D}(\mathbf{L})$ is $\mathrm{T}_{1}$ and moreover, it is compact (cf. Urquhart [14, Lemma 6]). If the lattice $\mathbf{L}$ is distributive, then $\mathcal{L}^{\mathrm{mp}}(\mathbf{L}, \underline{\mathbf{2}})=\mathcal{L}(\mathbf{L}, \underline{\mathbf{2}})$, the relation $E$ coincides with the pointwise partial order of maps and $\mathrm{D}(\mathbf{L})$ is the usual dual space of $\mathbf{L}$ in the Priestley duality [13].

Ploščica's representation of $\mathbf{L} \in \mathcal{L}$ is then obtained in the following way. One takes the family of continuous $E$-preserving partial maps from $\mathrm{D}(\mathbf{L})$ into $\underset{\mathcal{T}}{\mathbf{T}}$, where $E$ on $\underset{\sim}{\mathbf{2}}$ is taken to be the usual order. The maximally-defined members of this set are then shown to form a lattice isomorphic to $\mathbf{L}$, the isomorphism being given by the natural evaluation map $e_{\mathbf{L}}: \mathbf{L} \rightarrow \operatorname{ED}(\mathbf{L})$. We recall further details of the construction in Section 3.

Our strategy for obtaining the canonical extension $\mathbf{L}^{\delta}$ of $\mathbf{L}$ will be to replace $\mathrm{D}(\mathbf{L})$ above by $\mathrm{D}^{b}(\mathbf{L})$. Here $\mathrm{D}^{b}$ is the composition ${ }^{b} \circ \mathrm{D}$, where $b$ is the map forgetting the topology. Thus the category we shall use to build the canonical extension will be the category $\mathcal{G}$ of graphs $\mathbf{X}=(X, E)$ and partial maps which preserve $E$. (We warn that what we are calling a graph would usually be referred to as a digraph. We also warn that structures of the form $\mathrm{D}^{b}(\mathbf{L})$ will have special properties we shall need to exploit in due course.) Much of our work leading up to Theorem 3.11 involves checking that the ancillary results on the graphs with topology which underlie the Ploščica representation [12, Section 1] have appropriate analogues in the topologyfree setting.

Initially we let $\mathbf{X}=(X, E)$ be any graph. For two graphs $\mathbf{X}=\left(X, E_{X}\right)$ and $\mathbf{Y}=$ $\left(Y, E_{Y}\right)$ we use the notation $\mathcal{G}(\mathbf{X}, \mathbf{Y})$ to denote the collection of total $E$-preserving maps from $\mathbf{X}$ to $\mathbf{Y}$. In Section 4 we will make use of the set of partial morphisms, $\mathcal{G}^{\mathrm{p}}(\mathbf{X}, \mathbf{Y})$. We note that, by Zorn's Lemma, every partial $E$-preserving map into $\underset{\sim}{\mathbf{2}}$ can be extended to a maximal partial $E$-preserving map; by 'maximal' we mean here 
that there is no partial $E$-preserving map properly extending it (in general such an extension will not be unique). We will denote by $\mathcal{G}^{\mathrm{mp}}(\mathbf{X}, \underset{2}{\mathbf{2}})$ the set of maximal partial $E$-preserving maps from $\mathbf{X}$ to $\underset{\sim}{\mathbf{2}}$. Let us use the shorthand MPE to refer to an element of such a set.

In the distributive case we can restrict to the situation in which $E$ is a partial order and MPE's are simply total maps which are order-preserving. It is immediate that the order-preserving maps from a poset $\mathbf{X}$ into $\underset{\sim}{\mathbf{2}}$ always form a complete lattice under pointwise join and meet. In the case of MPE's on a graph the situation is more complicated.

We now work towards showing, for a general graph $\mathbf{X}=(X, E)$, that $\mathcal{G}^{\mathrm{mp}}(\mathbf{X}, \underset{\sim}{\mathbf{2}})$ does indeed form a complete lattice. As we shall see in Proposition 3.2 and the discussion preceding it, the similarity between Lemma 2.1 below and the corresponding result, [12, Lemma 1.3], concerning graphs with topology, is critical to the success of our approach to completions built from dual spaces. The key point is that in [12, Lemma 1.3] the topological assertions and those involving $E$ alone can be separated.

Lemma 2.1 (cf. [12, Lemma 1.3]) Let $\mathbf{X}=(X, E)$ be a graph and $\varphi \in \mathcal{G}^{\mathrm{mp}}(\mathbf{X}, \underset{\sim}{\mathbf{2}})$. Then

(i) $\varphi^{-1}(0)=\left\{x \in X \mid\right.$ there is no $y \in \varphi^{-1}$ (1) with $\left.(y, x) \in E\right\}$;

(ii) $\varphi^{-1}(1)=\left\{x \in X \mid\right.$ there is no $y \in \varphi^{-1}(0)$ with $\left.(x, y) \in E\right\}$.

Consider a family $\left\{\varphi_{i} \in \mathcal{G}^{\mathrm{mp}}(\mathbf{X}, \underset{\sim}{\mathbf{2}}) \mid i \in I\right\}$ for an index set $I$. Then we can define the pointwise meet $p_{\varphi_{i}}^{\wedge}$ and pointwise join $p_{\varphi_{i}}^{\vee}$ as follows:

$$
p_{\varphi_{i}}^{\wedge}(x)=\left\{\begin{array}{ll}
1 & \text { if } x \in \bigcap_{i \in I} \varphi_{i}^{-1}(1), \\
0 & \text { if } x \in \bigcup_{i \in I} \varphi_{i}^{-1}(0)
\end{array} \quad \text { and } \quad p_{\varphi_{i}}^{\vee}(x)= \begin{cases}1 & \text { if } x \in \bigcup_{i \in I} \varphi_{i}^{-1}(1), \\
0 & \text { if } x \in \bigcap_{i \in I} \varphi_{i}^{-1}(0)\end{cases}\right.
$$

(The motivation for these definitions comes from Ploščica [12, Section 3].) To see that these partial maps are $E$-preserving, let $\left(p_{\varphi_{i}}^{\wedge}(x), p_{\varphi_{i}}^{\wedge}(y)\right)=(1,0)$. Then there exists $j \in I$ with $\varphi_{j}(y)=0$, and thus $\left(\varphi_{j}(x), \varphi_{j}(y)\right)=(1,0)$ and so $(x, y) \notin E$.

We shall work with specific extensions of the maps $p_{\varphi_{i}}^{\wedge}$ and $p_{\varphi_{i}}^{\bigvee}$ to elements of $\mathcal{G}^{\mathrm{mp}}(\mathbf{X}, \underset{2}{\mathbf{2}})$. The motivation for our choice of maximal maps comes from Lemma 2.1. Define $\widetilde{e_{\varphi_{i}}}: \mathbf{X} \rightarrow \underset{\sim}{\mathbf{2}}$ and $e_{\varphi_{i}}^{\bigvee}: \mathbf{X} \rightarrow \underset{\sim}{\mathbf{2}}$ as follows:

$$
\begin{aligned}
& e_{\varphi_{i}}^{\wedge}(x)= \begin{cases}1 & \text { if } x \in \bigcap_{i \in I} \varphi_{i}^{-1}(1), \\
0 & \text { if there is no } y \in \bigcap_{i \in I} \varphi_{i}^{-1}(1) \text { with }(y, x) \in E ;\end{cases} \\
& e_{\varphi_{i}}^{\bigvee}(x)= \begin{cases}1 & \text { if there is no } y \in \bigcap_{i \in I} \varphi_{i}^{-1}(0) \text { with }(x, y) \in E, \\
0 & \text { if } x \in \bigcap_{i \in I} \varphi_{i}^{-1}(0) .\end{cases}
\end{aligned}
$$

We claim that $e_{\varphi_{i}}^{\wedge}$ extends $p_{\varphi_{i}}^{\wedge}$. Let $x \in \bigcup_{i \in I} \varphi_{i}^{-1}(0)$, so that $x \in \varphi_{j}^{-1}(0)$ for some $j \in I$. Then there is no $y \in \bigcap_{i \in I} \varphi_{i}^{-1}(1)$ with $(y, x) \in E$, for otherwise $\left(\varphi_{j}(y), \varphi_{j}(x)\right)=(1,0)$, which contradicts the preservation of $E$ by $\varphi_{j}$. Analogously, $e_{\varphi_{i}}^{\bigvee}$ extends $p_{\varphi_{i}}^{\bigvee}$. The next lemma shows that $e_{\varphi_{i}}^{\wedge}$ and $e_{\varphi_{i}}^{\vee}$ are maximal partial $E$-preserving extensions of $p_{\varphi_{i}}^{\wedge}$ and $p_{\varphi_{i}}$, respectively. 
Lemma 2.2 Let $\mathbf{X}=(X, E)$ be a graph and let $\left\{\varphi_{i} \mid i \in I\right\} \subseteq \mathcal{G}^{\mathrm{mp}}(\mathbf{X}, \underset{\sim}{\mathbf{2}})$. Then the maps $\hat{e}_{\varphi_{i}}^{\wedge}$ and $e_{\varphi_{i}}^{\vee}$ are elements of $\mathbf{G}^{\mathrm{mp}}(\mathbf{X}, \mathbf{2})$ extending $p_{\varphi_{i}}^{\wedge}$ and $p_{\varphi_{i}}^{\vee}$, respectively.

Proof To see that $e_{\varphi_{i}}^{\wedge}$ preserves $E$, consider $\left(e_{\varphi_{i}}^{\wedge}\left(x_{1}\right), e_{\varphi_{i}}^{\wedge}\left(x_{0}\right)\right)=(1,0)$. Then $x_{1} \in$ $\bigcap_{i \in I} \varphi_{i}^{-1}(1)$ and there is no $y \in \bigcap_{i \in I} \varphi_{i}^{-1}(1)$ with $\left(y, x_{0}\right) \in E$. Thus $\left(x_{1}, x_{0}\right) \notin E$ as required.

We now show the maximality of $e_{\varphi_{i}}^{\wedge}$. Let $\psi \in \mathcal{G}^{\mathrm{mp}}(\mathbf{X}, \underset{\sim}{\mathbf{2}})$ be a map extending $e_{\varphi_{i}}^{\wedge}$. Let $x \in X$ be such that $x \notin \operatorname{dom}\left(\hat{e}_{\varphi_{i}}\right)$; thus $x \notin \bigcap_{i \in I} \varphi_{i}^{-1}(1)$ and there is $y_{1} \in$ $\bigcap_{i \in I} \varphi_{i}^{-1}(1)$ with $\left(y_{1}, x\right) \in E$. We want to show that $x \notin \operatorname{dom}(\psi)$. Let $j \in I$ be such that $x \notin \varphi_{j}^{-1}(1)$. Since $\varphi_{j} \in \mathcal{G}^{\mathrm{mp}}(\mathbf{X}, \underset{\sim}{\mathbf{2}})$, by Lemma 2.1 there exists $y_{0} \in \varphi_{j}^{-1}(0)$ with $\left(x, y_{0}\right) \in E$. Hence $\varphi_{j}\left(y_{0}\right)=0$ and so, by Lemma 2.1 again, there is no $y \in \varphi_{j}^{-1}(1)$ with $\left(y, y_{0}\right) \in E$. Hence there is no $y \in \bigcap_{i \in I} \varphi_{i}^{-1}(1)$ with $\left(y, y_{0}\right) \in E$, which gives us $e_{\varphi_{i}}^{\wedge}\left(y_{0}\right)=0$. As $\psi$ extends $e_{\varphi_{i}}^{\wedge}$, we obtain $\psi\left(y_{0}\right)=0$. Moreover, as $\hat{e}_{\varphi_{i}}^{\wedge}\left(y_{1}\right)=1$, we have $\psi\left(y_{1}\right)=1$.

Now suppose for a contradiction that $x \in \operatorname{dom}(\psi)$. Then $\left(y_{1}, x\right) \in E$ and $\left(x, y_{0}\right) \in E$ give us $1=\psi\left(y_{1}\right) \leqslant \psi(x) \leqslant \psi\left(y_{0}\right)=0$, which is false.

Theorem 2.3 Let $\mathbf{X}=(X, E)$ be a graph. Then the set $\mathrm{C}(\mathbf{X})=\mathcal{G}^{\mathrm{mp}}(\mathbf{X}, \underset{\sim}{\mathbf{2}})$ ordered by the rule

$$
\varphi \leqslant \psi \Longleftrightarrow \varphi^{-1}(1) \subseteq \psi^{-1}(1)
$$

is a complete lattice.

Proof Obviously, the relation $\leqslant$ is reflexive and transitive. The antisymmetry of $\leqslant$ follows from the fact that for any $\varphi, \psi \in \mathbf{G}^{\mathrm{mp}}(\mathbf{X}, \underset{\sim}{\mathbf{2}})$, if $\varphi^{-1}(1)=\psi^{-1}(1)$, then, by Lemma 2.1, $\varphi^{-1}(0)=\psi^{-1}(0)$, and hence $\varphi=\psi$.

To show that $\leqslant$ is a (complete) lattice order, let $\left\{\varphi_{i} \mid i \in I\right\} \subseteq \mathcal{G}^{\mathrm{mp}}(\mathbf{X}, \underset{\sim}{\mathbf{2}})$. We claim that $\bigwedge \varphi_{i}=e_{\varphi_{i}}^{\wedge}$ which is an element of $\mathrm{C}(\mathbf{X})$ by Lemma 2.2.

First note that $\left(e_{\varphi_{i}}^{\wedge}\right)^{-1}(1)=\bigcap_{i \in I} \varphi_{i}^{-1}(1) \subseteq \varphi_{i}^{-1}(1)$ for all $i \in I$. Thus in $(\mathrm{C}(\mathbf{X}), \leqslant)$, we have $e_{\varphi_{i}}^{\wedge} \leqslant \varphi_{i}$ for all $i \in I$. Now let $\psi \in \mathcal{G}^{\mathrm{mp}}(\mathbf{X}, \underset{\sim}{\mathbf{2}})$ such that $\psi \leqslant \varphi_{i}$ for all $i \in I$. Then $\psi^{-1}(1) \subseteq\left(\bigcap_{i \in I} \varphi_{i}\right)^{-1}(1)=\left(e_{\varphi_{i}}^{\wedge}\right)^{-1}(1)$, whence $\psi \leqslant e_{\varphi_{i}}^{\wedge}$ as required.

Before we move on to incorporate topology we insert a lemma (the proof of which is straightforward) concerning maps between graphs. We shall need this in Section 4, when we consider morphisms acting by composition.

Lemma 2.4 Let $\mathbf{X}=\left(X, E_{X}\right)$ and $\mathbf{Y}=\left(Y, E_{Y}\right)$ be graphs and let $\alpha \in \mathcal{G}(\mathbf{X}$, Y). Then for $\varphi \in \mathcal{G}^{\mathrm{P}}(\mathbf{Y}, \mathbf{2})$, the map $\varphi \circ \alpha$, with the domain being determined by the set of elements for which the composition is defined, is a partial map from $\mathbf{X}$ to $\underset{\sim}{\mathbf{2}}$ which preserves $E$.

A set equipped with a reflexive binary relation and a topology will be called a graph with topology. We make the family $\mathcal{G}_{\mathcal{T}}$ of graphs with topology into a category in the following way. A map $\varphi:\left(X_{1}, E_{1}, \tau_{1}\right) \rightarrow\left(X_{2}, E_{2}, \tau_{2}\right)$ between graphs with topology is called a $\mathcal{G}_{\mathcal{T}}$-morphism if it preserves the binary relation and is continuous as a map from $\left(X_{1}, \tau_{1}\right)$ to $\left(X_{2}, \tau_{2}\right)$. A partial map $\varphi:\left(X_{1}, E_{1}, \tau_{1}\right) \rightarrow\left(X_{2}, E_{2}, \tau_{2}\right)$ 
is called a partial $\mathcal{G}_{\mathcal{T}}$-morphism if its domain is a $\tau_{1}$-closed subset of $X_{1}$ and the restriction of $\varphi$ to its domain is a morphism. (We assume that $\operatorname{dom}(\varphi)$ inherits the binary relation and the topology from $X_{1}$.) A partial $\mathcal{G}_{\mathcal{T}}$-morphism is called maximal, or an MPM for short, if there is no partial $\mathcal{G}_{\mathcal{T}}$-morphism properly extending it. For a graph with topology $\mathbf{X}_{\mathcal{T}}=(X, E, \mathcal{T})$ we denote by $\mathcal{G}_{\mathcal{T}}^{\mathrm{mp}}\left(\mathbf{X}_{\mathcal{T}},{\underset{\sim}{\mathcal{T}}}_{\mathcal{T}}\right)$ the set of MPM's from $\mathbf{X}_{\mathcal{T}}$ to $\underset{\mathcal{2}}{\mathbf{2}}$.

Our candidate for the canonical extension of a bounded lattice $\mathbf{L}$ is the complete lattice $\mathcal{G}^{\mathrm{mp}}\left(\mathrm{D}^{\mathrm{b}}(\mathbf{L}), \underset{\sim}{\mathbf{2}}\right)=\mathrm{C}\left(\mathrm{D}^{\mathrm{b}}(\mathbf{L})\right)$. The embedding will be given, as in the Ploščica representation (see Proposition 3.1 below) by the map $e_{\mathbf{L}}$, given by evaluation, onto the set of maps which are maximal among continuous partial morphisms into $\underset{\sim}{\mathbf{2}} \mathcal{T}$. It is therefore necessary to reconcile the two versions of maximality-one with topology and the other without. This can be done for graphs in general, rather than just those arising from dual spaces.

Proposition 2.5 Let $\mathbf{X}_{\mathcal{T}}=(X, E, \mathcal{T})$ be a graph with topology and $\mathbf{X}=(X, E)$ be its untopologised counterpart. Then $\boldsymbol{G}_{\mathcal{T}}^{\mathrm{mp}}\left(\mathbf{X}_{\mathcal{T}}, \underset{\sim}{\mathbf{2}}\right) \subseteq \mathcal{G}^{\mathrm{mp}}(\mathbf{X}, \underset{\sim}{\mathbf{2}})$.

Proof We have already observed that Lemma 2.1 has a topological counterpart shown in [12, Lemma 1.3]. Specifically, for $\varphi \in \mathbf{G}_{\mathcal{T}}^{\mathrm{mp}}\left(\mathbf{X}_{\mathcal{T}}, \underset{\sim}{\mathbf{2}_{\mathcal{T}}}\right)$ we have

$$
\begin{aligned}
& \varphi^{-1}(0)=\left\{x \in X \mid \text { there is no } y \in \varphi^{-1}(1) \text { with }(y, x) \in E\right\} \\
& \varphi^{-1}(1)=\left\{x \in X \mid \text { there is no } y \in \varphi^{-1}(0) \text { with }(x, y) \in E\right\} .
\end{aligned}
$$

Comparing the above with the properties of $\mathcal{G}^{\mathrm{mp}}(\mathbf{X}, \underset{\sim}{\mathbf{2}})$ in Lemma 2.1 we assert that any $\varphi \in \mathcal{G}_{\mathcal{T}}^{\mathrm{mp}}\left(\mathbf{X}_{\mathcal{T}},{\underset{\sim}{\mathcal{T}}}_{\mathcal{T}}\right)$ is maximal as a partial $E$-preserving map when the topology is suppressed. To verify this, suppose for a contradiction that $\psi \in \mathcal{G}^{\mathrm{mp}}(\mathbf{X}, \underset{\sim}{\mathbf{2}})$ strictly extends $\varphi \in \mathbf{G}_{\mathcal{T}}^{\mathrm{mp}}\left(\mathbf{X}_{\mathcal{T}}, \underset{\sim}{\mathbf{2}}\right)$. Let $x \in \operatorname{dom}(\psi) \backslash \operatorname{dom}(\varphi)$ and assume without loss of generality that $\psi(x)=1$. By maximality of $\psi$, Lemma 2.1 implies that there is no $y \in \operatorname{dom}(\psi)$ such that $\psi(y)=0$ and $(x, y) \in E$. In particular, there is no $y \in \operatorname{dom}(\varphi)$ such that $\varphi(y)=0$ and $(x, y) \in E$. Now, by the characterisation of $\varphi^{-1}(1)$ for $\varphi \in \mathcal{G}_{\mathcal{T}}^{\mathrm{mp}}\left(\mathbf{X}_{\mathcal{T}}, \mathbf{\sim}_{\mathcal{T}}\right)$, we deduce that $x \in \varphi^{-1}(1)$, which is the required contradiction.

The set $\mathcal{G}_{\mathcal{T}}^{\mathrm{mp}}\left(\mathbf{X}_{\mathcal{T}},{\underset{\sim}{\boldsymbol{2}}}_{\mathcal{T}}\right)$ can be considered as a subposet of the poset $\mathcal{G}^{\mathrm{mp}}(\mathbf{X}, \underset{\sim}{\mathbf{2}})$ with the partial order on $\widetilde{\mathcal{G}}_{\mathcal{T}}^{\mathrm{mp}}\left(\mathbf{X}_{\mathcal{T}}, \underset{\sim}{\mathbf{2}}\right)$ given, as in [12], by

$$
\varphi \leqslant \psi \quad \text { iff } \varphi^{-1}(1) \subseteq \psi^{-1}(1) .
$$

If in particular $\mathbf{X}_{\mathcal{T}}=\mathrm{D}(\mathbf{L})$ then it was shown in [12] (see Proposition 3.1(iii) below) that the partial order $\leqslant$ on $\mathcal{G}_{\mathcal{T}}^{\mathrm{mp}}\left(\mathbf{X}_{\mathcal{T}}, \underset{\sim}{\mathbf{2}} \mathcal{T}\right)$ is a lattice order, and the lattice $\left(\boldsymbol{G}_{\mathcal{T}}^{\mathrm{mp}}\left(\mathbf{X}_{\mathcal{T}}, \underset{\sim}{\boldsymbol{2}}\right), \leqslant\right)$ is clearly a sublattice of the lattice $\mathrm{C}(\mathbf{X})=\left(\boldsymbol{G}^{\mathrm{mp}}(\mathbf{X}, \underset{\sim}{\mathbf{2}}), \leqslant\right)$ (see Theorem 2.3).

\section{Canonical Extensions Constructed via Ploščica's Representation}

Our next task is to establish that, when we take a bounded lattice $\mathbf{L}$ and the graph $\mathbf{X}=\mathrm{D}^{b}(\mathbf{L})$, then $\mathrm{C}(\mathbf{X})$ acts as a completion of $\mathbf{L}$. For this we call on the Ploščica 
representation, and so now need to recall further facts from [12]. The relationship between a lattice $\mathbf{L}$ and the set $\mathcal{G}_{\mathcal{T}}^{\mathrm{mp}}\left(\mathrm{D}(\mathbf{L}), \mathbf{\sim}_{\mathcal{T}}\right)$ is summed up in the following result.

Proposition 3.1 [12, Lemmas 1.2 and 1.5 and Theorem 1.7] Let $\mathbf{L} \in \mathcal{L}$ and let $\mathrm{D}(\mathbf{L})$ be the graph with topology defining the dual space of $\mathbf{L}$. For $a \in L$, let the evaluation map $e_{a}: \mathrm{D}(\mathbf{L}) \rightarrow \underset{\sim}{\mathbf{2}}$ be defined by

$$
e_{a}(f)= \begin{cases}f(a) & a \in \operatorname{dom}(f), \\ - & \text { undefined otherwise. }\end{cases}
$$

Then the following hold.

(i) The map $e_{a} \in \mathcal{G}_{\mathcal{T}}^{\mathrm{mp}}\left(\mathrm{D}(\mathbf{L}), \mathbf{\sim}_{\mathcal{T}}\right)$ for each $a \in L$.

(ii) Every $\varphi \in \mathcal{G}_{\mathcal{T}}^{\mathrm{mp}}\left(\mathrm{D}(\mathbf{L}), \mathbf{2}_{\mathcal{T}}\right)$ is of the form $e_{a}$ for some $a \in L$.

(iii) The map $e_{\mathbf{L}}: \mathbf{L} \rightarrow \mathcal{G}_{\mathcal{T}}^{m \vec{p}}\left(\mathbf{D}(\mathbf{L}),{\underset{\sim}{\mathcal{T}}}_{\mathcal{T}}\right)$ given by evaluation, $a \mapsto e_{a}(a \in L)$, is an isomorphism of $\mathbf{L}$ onto the lattice $\mathcal{G}_{\mathcal{T}}^{\mathrm{mp}}\left(\mathbf{D}(\mathbf{L}), \mathbf{\sim}_{\mathcal{T}}\right)$, ordered by $\varphi \leqslant \psi$ if and only if $\varphi^{-1}(1) \subseteq \psi^{-1}(1)$.

By combining preceding results we obtain the following proposition.

Proposition 3.2 Let $\mathbf{L} \in \mathcal{L}$ and let $\mathrm{D}(\mathbf{L})$ be the graph with topology dual to $\mathbf{L}$ and let $\mathbf{X}=\mathrm{D}^{b}(\mathbf{L})$. Then $(e, \mathrm{C}(\mathbf{X}))$ is a completion of $\mathbf{L}$, where $e: a \mapsto e_{a}(a \in L)$.

Proof Theorem 2.3 tells us that $\mathrm{C}(\mathbf{X})$ is a complete lattice. The result now follows directly from Proposition 3.1 combined with the fact that every $\varphi \in \mathcal{G}_{\mathcal{T}}^{\mathrm{mp}}\left(\mathbf{D}(\mathbf{L}), \mathbf{\sim}_{\mathcal{T}}\right)$, and in particular any evaluation map $e_{a}$, belongs to $\mathcal{G}^{\mathrm{mp}}\left(\mathrm{D}^{\mathrm{b}}(\mathbf{L}), \underset{\sim}{\mathbf{2}}\right)$, by Proposition 2.5.

Proposition 3.2 identifies a completion for any bounded lattice constructed from the dual space of the lattice. When the lattice is distributive this certainly does give the canonical extension as introduced by Gehrke and Jónsson [8]. We would now like to prove that this completion supplies a canonical extension for an arbitrary bounded lattice. To achieve this, we need to examine more closely the structure of the dual space $\mathrm{D}(\mathbf{L})$ of a bounded lattice $\mathbf{L}$.

In [12, Section 2], Ploščica demonstrates how his dual representation for lattices relates to Urquhart's topological representation [14]. At the level of the dual spaces, the passage back and forth between Urquhart's dual representation and Ploščica's is set up by a bijection between maximal disjoint filter-ideal pairs in $\mathbf{L}$ (as employed by Urquhart) and $\mathcal{L}^{\mathrm{mp}}(\mathbf{L}, \underline{\mathbf{2}})$. Instead of carrying a single binary relation $E$, Urquhart's dual spaces are equipped with a pair of quasi-orders, $\leqslant_{1}$ and $\leqslant_{2}$. Interpreted in terms of MPH's, these two relations are defined on the set $\mathcal{L}^{\mathrm{mp}}(\mathbf{L}, \underline{\mathbf{2}})$ as follows:

$$
f \leqslant_{1} g \Longleftrightarrow f^{-1}(1) \subseteq g^{-1}(1) \text { and } f \leqslant_{2} g \Longleftrightarrow f^{-1}(0) \subseteq g^{-1}(0) .
$$

These quasi-orders $\leqslant_{1}$ and $\leqslant_{2}$ prove to be a valuable ancillary tool for working with graphs of the form $\mathrm{D}^{\mathrm{b}}(\mathbf{L})=\left(\mathcal{L}^{\mathrm{mp}}(\mathbf{L}, \underline{\mathbf{2}}), E\right)$, and we shall use them, in an analogous manner but on bigger domains, in Section 4.

Lemma 3.3 [12, Theorem 2.1] Let $\mathbf{L} \in \mathcal{L}$ and let $f, g \in \mathcal{L}^{\mathrm{mp}}(\mathbf{L}, \underline{\mathbf{2}})$. Then

(i) $(f, g) \in E$ if and only if there exists $h \in \mathcal{L}^{\mathrm{mp}}(\mathbf{L}, \underline{\mathbf{2}})$ with $f \leqslant_{1} h$ and $g \leqslant_{2} h$; 
(ii) $f \leqslant_{2} g$ if and only if there is no $h \in \mathcal{L}^{\mathrm{mp}}(\mathbf{L}, \underline{\mathbf{2}})$ with $(h, g) \in E$ and $(h, f) \notin E$;

(iii) $f \leqslant_{1} g$ if and only if there is no $h \in \mathcal{L}^{\mathrm{mp}}(\mathbf{L}, \underline{\mathbf{2}})$ with $(g, h) \in E$ and $(f, h) \notin E$.

It is a consequence of (i) above that $\leqslant_{1} \subseteq E$ and $\geqslant_{2} \subseteq E$. This will be used repeatedly below.

Lemma 3.4 Let $\mathbf{L} \in \mathcal{L}$. Let $\mathbf{X}=\mathrm{D}^{b}(\mathbf{L})$ and let $\varphi \in \mathcal{G}^{\mathrm{mp}}(\mathbf{X}, \underset{\sim}{\mathbf{2}})$. Then

(i) if $f \notin \varphi^{-1}(0)$ there exists $g \in \varphi^{-1}(1)$ such that $f \leqslant 2 g$;

(ii) if $f \notin \varphi^{-1}(1)$ there exists $g \in \varphi^{-1}(0)$ such that $f \leqslant 1 g$.

Proof (i) Given $f \notin \varphi^{-1}(0)$, we have by Lemma 2.1 that there exists $h \in \varphi^{-1}$ (1) such that $(h, f) \in E$. By Lemma 3.3(i) there exists $g \in X$ such that $h \leqslant_{1} g$ and $f \leqslant 2 g$, and we now claim that $g \in \varphi^{-1}(1)$. If we suppose that $g \notin \varphi^{-1}(1)$, by Lemma 2.1 there must exist $u \in \varphi^{-1}(0)$ such that $(g, u) \in E$. Now $(g, u) \in E$ if and only if there exists $v \in X$ such that $g \leqslant_{1} v$ and $u \leqslant_{2} v$, again by Lemma 3.3(i). By the transitivity of $\leqslant_{1}$ we have that $h \leqslant_{1} v$. Applying this with $u \leqslant_{2} v$ gives $(h, u) \in E$. Since $u \in \varphi^{-1}(0)$ and $h \in \varphi^{-1}(1)$, this contradicts that $\varphi$ is $E$-preserving. Thus $g \in \varphi^{-1}(1)$. The proof of (ii) is similar.

Lemma 3.5 Let $\mathbf{L} \in \mathcal{L}, \mathbf{X}=\mathrm{D}^{b}(\mathbf{L})$ and $\varphi \in \mathcal{G}^{\mathrm{mp}}(\mathbf{X}, \underset{\sim}{\mathbf{2}})$. For $f, g \in \mathcal{L}^{\mathrm{mp}}(\mathbf{L}, \underline{\mathbf{2}})$

(i) if $f \leqslant_{2} g$ and $\varphi(f)=0$, then $\varphi(g)=0$;

(ii) if $f \leqslant_{1} g$ and $\varphi(f)=1$, then $\varphi(g)=1$.

Proof Let $f \leqslant_{2} g$ and $\varphi(f)=0$ and suppose that $g \notin \varphi^{-1}(0)$. Then by Lemma 3.4(i) there exists $h \in \varphi^{-1}(1)$ such that $g \leqslant_{2} h$. By the transitivity of $\leqslant_{2}$, we then see that $f \leqslant_{2} h$ and so $(h, f) \in E$. Now $\varphi(h)=1 \nless 0=\varphi(f)$, contradicting the fact that $\varphi$ is $E$-preserving. Thus $\varphi(g)=0$.

We can now show that $\mathcal{G}^{\mathrm{mp}}\left(\mathrm{D}^{\mathrm{b}}(\mathbf{L}), \underset{\sim}{\mathbf{2}}\right)$, as an extension of $\boldsymbol{G}_{\mathcal{T}}^{\mathrm{mp}}(\mathrm{D}(\mathbf{L}), \underset{\sim}{\mathbf{2}})$, satisfies the density condition. That is, every element of $\mathcal{G}^{\mathrm{mp}}\left(\mathrm{D}^{\mathrm{b}}(\mathbf{L}), \underset{\sim}{\mathbf{2}}\right)$ can be written as both a join of meets and a meet of joins of elements of $\mathcal{G}_{\mathcal{T}}^{\mathrm{mp}}(\mathrm{D}(\mathbf{L}), \underset{\sim}{\mathbf{2}})$.

Proposition 3.6 (Density) Let $\mathbf{L} \in \mathcal{L}$ and let $\mathbf{X}=\mathrm{D}^{\mathrm{b}}(\mathbf{L})$. Then every element $\varphi$ of the completion $\mathrm{C}(\mathbf{X})=\mathcal{G}^{\mathrm{mp}}\left(\mathrm{D}^{\mathrm{b}}(\mathbf{L}), \underset{\sim}{\mathbf{2}}\right)$ can be expressed as

$$
\varphi=\bigvee\{\bigwedge F \mid \bigwedge F \leqslant \varphi\}=\bigwedge\{\bigvee I \mid \varphi \leqslant \bigvee I\}
$$

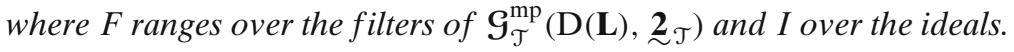

Proof If we let $\psi=\bigvee\{\bigwedge F \mid \bigwedge F \leqslant \varphi\}$, it is clear that $\psi \leqslant \varphi$. In order to show that $\varphi \leqslant \psi$, we must check that $\varphi^{-1}(1) \subseteq \psi^{-1}(1)$. Therefore, using the definitions of joins and meets in $\mathcal{G}^{\mathrm{mp}}\left(\mathrm{D}^{b}(\mathbf{L}), \underset{\sim}{\mathbf{2}}\right)$ in Lemma 2.2, and given $x \in \varphi^{-1}(1)$, we must show that if $y \in \bigcap\left\{(\bigwedge F)^{-1}(0) \mid \bigwedge F \leqslant \varphi\right\}$, then $(x, y) \notin E$.

In order to do this, we claim that $\bigcap\left\{(\bigwedge F)^{-1}(0) \mid \bigwedge F \leqslant \varphi\right\} \subseteq \varphi^{-1}(0)$. Suppose that $f \notin \varphi^{-1}(0)$. By Lemma 3.4(i) there exists $g \in \varphi^{-1}(1)$ such that $f \leqslant 2 g$. Now we consider $\mathscr{F}_{g}:=\{a \in L \mid g(a)=1\}$, a filter of $\mathbf{L}$. From Proposition 3.1(iii), we can see that a set $F \subseteq \mathcal{G}_{\mathcal{T}}^{\mathrm{mp}}\left(\mathrm{D}(\mathbf{L}),{\underset{\sim}{\mathcal{T}}}_{\mathcal{T}}\right)$ is a filter if and only if $F=\left\{e_{b} \mid b \in \mathscr{F}\right\}$ where $\mathscr{F}$ is a 
filter of $L$. This gives us a filter $G$ of $\mathcal{G}_{\mathcal{T}}^{\mathrm{mp}}(\mathrm{D}(\mathbf{L}), \underset{\mathcal{T}}{\mathbf{2}})$, with $G=\left\{e_{a} \mid a \in \mathscr{F}_{g}\right\}$, and we will show that $\bigwedge G \leqslant \varphi$. If $h \in(\bigwedge G)^{-1}(1)$, then $h \in \bigcap\left\{e_{a}^{-1}(1) \mid a \in \mathscr{F}_{g}\right\}$. That is, for every $a \in L$ such that $g(a)=1, h(a)=1$. So $g^{-1}(1) \subseteq h^{-1}(1)$, giving us that $g \leqslant_{1} h$. Now we use Lemma 3.5(ii) to get $\varphi(h)=1$ and hence $(\bigwedge G)^{-1}(1) \subseteq \varphi^{-1}(1)$.

We have $\bigwedge G \leqslant \varphi$ and now show that $f \notin(\bigwedge G)^{-1}(0)$. By definition

$$
(\bigwedge G)^{-1}(0)=\left\{b \in \mathcal{L}^{\mathrm{mp}}(\mathbf{L}, \underline{\mathbf{2}}) \mid \text { for all } c \in \bigcap\left\{e_{a}^{-1}(1) \mid a \in \mathscr{F}_{g}\right\},(c, b) \notin E\right\}
$$

and clearly $g \in \bigcap\left\{e_{a}^{-1}(1) \mid a \in \mathscr{F}_{g}\right\}$. Now, our original assumption that $f \leqslant 2 g$ gives us that $(g, f) \in E$ and so $f \notin(\bigwedge G)^{-1}(0)$. Hence $f \notin \bigcap\left\{(\bigwedge F)^{-1}(0) \mid \bigwedge F \leqslant \varphi\right\}$ and thus we have $\bigcap\left\{(\bigwedge F)^{-1}(0) \mid \bigwedge F \leqslant \varphi\right\} \subseteq \varphi^{-1}(0)$.

Now recall that $x \in \varphi^{-1}(1)$ and let $y \in \bigcap\left\{(\bigwedge F)^{-1}(0) \mid \bigwedge F \leqslant \varphi\right\}$. Then $(x, y) \in E$ would imply $\varphi(x)=1 \nless 0=\varphi(y)$, contradicting $\varphi$ being $E$-preserving. Thus $(x, y) \notin E$ and we have $x \in \psi^{-1}(1)$ as required.

In Proposition 3.6 we proved very directly that $\mathrm{C}\left(\mathrm{D}^{\mathrm{b}}(\mathbf{L})\right)$ supplies a dense completion of $\mathbf{L}$. We now want to prove that this completion is compact. This is true, but not entirely straightforward to prove. We shall go via a somewhat circuitous route, obtaining on the way characterisations of filter and ideal elements in $C\left(D^{b}(\mathbf{L})\right)$ which are of independent interest.

To describe the filter and ideal elements of the extension $C\left(D^{b}(\mathbf{L})\right)$ we need to look more closely at the topology on $\mathrm{D}(\mathbf{L})=\left(\mathcal{L}^{\mathrm{mp}}(\mathbf{L}, \underline{\mathbf{2}}), E, \mathcal{T}\right)$. We recall that $\mathcal{T}$ has subbasic closed sets of the form $V_{a}=\left\{f \in \mathcal{L}^{\mathrm{mp}}(\mathbf{L}, \underline{\mathbf{2}}) \mid f(a)=0\right\}$ and $W_{a}=$ $\left\{f \in \mathcal{L}^{\mathrm{mp}}(\mathbf{L}, \underline{\mathbf{2}}) \mid f(a)=1\right\}$ for $a \in L$. We observe that from Lemma 3.5, for any $\varphi \in \mathcal{G}^{\mathrm{mp}}\left(\mathrm{D}^{\mathrm{b}}(\mathbf{L}), \underset{\sim}{\mathbf{2}}\right)$, we have that $\varphi^{-1}(1)$ is a $\leqslant_{1}$-increasing set, and $\varphi^{-1}(0)$ is a $\leqslant_{2^{-}}$ increasing set. We also note that the intersection of a collection of closed sets of the form $W_{b}$ for $b \in L$ will be $\leqslant{ }_{1}$-increasing, while the intersection of sets of the form $V_{a}$ with $a \in L$ will be $\leqslant_{2}$-increasing.

The following result parallels [12, Lemma 1.4] though our approach in proving it is different.

Lemma 3.7 Let $\mathbf{L} \in \mathcal{L}$ and let $a \in$ L. Let $\mathbf{X}=\mathrm{D}^{b}(\mathbf{L})$ and let $\varphi \in \mathcal{G}^{\mathrm{mp}}\left(\mathrm{D}^{b}(\mathbf{L}), \underset{\sim}{\mathbf{2}}\right)$. Then

(i) $\varphi^{-1}(0) \cap W_{a}=\emptyset$ implies $\varphi^{-1}(0) \subseteq V_{a}$;

(ii) $\varphi^{-1}(1) \cap V_{a}=\emptyset$ implies $\varphi^{-1}(1) \subseteq W_{a}$.

Proof We consider (i). Let $\varphi^{-1}(0) \cap W_{a}=\emptyset$. Suppose that $f \in \varphi^{-1}(0)$ and $f \notin V_{a}$. As $V_{a}=e_{a}^{-1}(0)$, by Lemma 3.4(i) there exists $g \in \varphi^{-1}(1)$ such that $f \leqslant 2 g$. Since $\varphi(f)=0$, by Lemma 3.5(i) we obtain $\varphi(g)=0$, a contradiction.

Lemma 3.8 Let $\mathbf{L} \in \mathcal{L}$, let $\mathbf{X}_{\mathcal{T}}=\mathrm{D}(\mathbf{L})$ and let $\varphi$ be an element of $\mathcal{G}^{\mathrm{mp}}\left(\mathrm{D}^{\mathrm{b}}(\mathbf{L})\right.$, $\left.\underset{\sim}{\mathbf{2}}\right)$. Then

(i) $\varphi^{-1}(1)$ is a $\mathcal{T}$-closed subset of $\mathbf{X}_{\mathcal{T}}$ if and only if $\varphi^{-1}(1)=\bigcap\left\{W_{b} \mid b \in K\right\}$, for some $K \subseteq L$;

(ii) $\varphi^{-1}(0)$ is a $\mathcal{T}$-closed subset of $\mathbf{X}_{\mathcal{T}}$ if and only if $\varphi^{-1}(0)=\bigcap\left\{V_{a} \mid a \in M\right\}$, for some $M \subseteq L$. 
Proof We consider (i) and so let $X$ denote the underlying set of $\mathbf{X}_{\mathcal{T}}$, that is, $X=$ $\mathcal{L}^{\mathrm{mp}}(\mathbf{L}, \underline{\mathbf{2}})$. The sufficiency is clear as each $W_{b}$ is $\mathcal{T}$-closed. For the necessity, let $\varphi^{-1}(1)$ be a $\mathcal{T}$-closed subset of $\mathbf{X}_{\mathcal{T}}$. Let

$$
\mathcal{S}:=\left\{W_{b} \mid b \in L, \varphi^{-1}(1) \subseteq W_{b}\right\}
$$

be the family of all sets $W_{b}$ containing $\varphi^{-1}(1)$ and $K:=\left\{b \in L \mid W_{b} \in \mathcal{S}\right\}$. As $\varphi^{-1}(1) \subseteq W_{b}$ for each $b \in K$, it is obvious that $\varphi^{-1}(1) \subseteq \bigcap\left\{W_{b} \mid b \in K\right\}$. The reverse inclusion will hold too provided we can show that $f \notin \varphi^{-1}(1)$ implies the existence of $W_{b} \in \mathcal{S}$ such that $f \notin W_{b}$.

Let $f \notin \varphi^{-1}(1)$. By Lemma 2.1(ii), there exists $g \in \varphi^{-1}(0)$ such that $(f, g) \in E$. Since $g \in \varphi^{-1}(0)$, for every $h \in \varphi^{-1}(1)$ we necessarily have $(h, g) \notin E$. By definition of the relation $E$ this means that there exists $b_{h} \in L$ such that $h\left(b_{h}\right)=1$ and $g\left(b_{h}\right)=0$. We note that each set $X \backslash V_{b_{h}}$ is $\mathcal{T}$-open and $h \in X \backslash V_{b_{h}}$. Hence we have that $\varphi^{-1}(1) \subseteq \bigcup\left\{X \backslash V_{b_{h}} \mid h \in \varphi^{-1}(1)\right\}$. Since the space $\mathbf{X}_{\mathcal{T}}$ is compact and $\varphi^{-1}(1)$ is a $\mathcal{T}$ closed subset of $\mathbf{X}_{\mathcal{T}}$ by hypothesis, there are $b_{1}, \ldots, b_{n} \in L$ such that $g\left(b_{1}\right)=\cdots=$ $g\left(b_{n}\right)=0$ and $\varphi^{-1}(1) \subseteq\left(X \backslash V_{b_{1}}\right) \cup \cdots \cup\left(X \backslash V_{b_{n}}\right)$. Let $b:=b_{1} \vee \cdots \vee b_{n} \in L$. We have $g(b)=0$ and $\varphi^{-1}(1) \subseteq X \backslash V_{b}$. The latter by Lemma 3.7 yields $\varphi^{-1}(1) \subseteq W_{b}$, and thus $W_{b} \in \mathcal{S}$. Since $(f, g) \in E$ and $g(b)=0$, we cannot have $f(b)=1$. Hence $f \notin W_{b}$, as required.

Proposition 3.9 (Filter and ideal elements) Let $\mathbf{L} \in \mathcal{L}, \mathbf{X}_{\mathcal{T}}=\mathrm{D}(\mathbf{L})$ and let $\varphi$ be an element of $\mathcal{G}^{\mathrm{mp}}\left(\mathrm{D}^{\mathrm{b}}(\mathbf{L}), \underset{\sim}{\mathbf{2}}\right)=\mathrm{C}(\mathbf{X})$. Then the three conditions in (i) are equivalent and the three conditions in (ii) are equivalent:

(i) (1) $\varphi$ is a filter element of $\mathrm{C}(\mathbf{X})$;

(2) $\varphi^{-1}(1)=\bigcap\left\{W_{b} \mid b \in K\right\}$, for some $K \subseteq L$;

(3) $\varphi^{-1}(1)$ is a $\mathcal{T}$-closed subset of $\mathbf{X}_{\mathcal{T}}$.

(ii) (1) $\varphi$ is an ideal element of $\mathrm{C}(\mathbf{X})$;

(2) $\varphi^{-1}(0)=\bigcap\left\{V_{a} \mid a \in M\right\}$, for some $M \subseteq L$;

(3) $\varphi^{-1}(0)$ is a $\mathcal{T}$-closed subset of $\mathbf{X}_{\mathcal{T}}$.

Proof The equivalences of (2) and (3) in (i) and (ii) come from Lemma 3.8. Now let (1) in (i) hold, that is, let $\varphi$ be a filter element of $\mathrm{C}(\mathbf{X})$. Then we have that $\varphi=\bigwedge \psi_{i}$ where $\left\{\psi_{i} \mid i \in I\right\} \subseteq \mathcal{G}_{\mathcal{T}}^{\mathrm{mp}}\left(\mathrm{D}(\mathbf{L}),{\underset{\sim}{\mathcal{T}}}_{\mathcal{T}}\right)$ for some index set $I$. From the representation theorem (see Proposition 3.1), we know that each $\psi_{i}$ is in fact $e_{b_{i}}$ for some $b_{i} \in L$ and from the definition of a meet of elements of $\mathcal{G}^{\mathrm{mp}}\left(\mathrm{D}^{\mathrm{b}}(\mathbf{L}), \underset{\sim}{\mathbf{2}}\right)$, we have

$$
\varphi^{-1}(1)=\bigcap_{i \in I} \psi_{i}^{-1}(1)=\bigcap_{i \in I} e_{b_{i}}^{-1}(1)=\bigcap_{i \in I} W_{b_{i}} .
$$

Hence (1) implies (2). Now let (2) hold. Then $\varphi^{-1}(1)=\bigcap_{i \in I} e_{b_{i}}^{-1}(1)$, and thus $\varphi^{-1}(1)$ can be expressed as the meet of images of lattice elements in $C(\mathbf{X})$, yielding (1). The equivalence of (1) and (2) in (ii) can be shown analogously.

We are now able to prove that our completion is indeed a compact completion.

Proposition 3.10 (Compactness) Let $\mathbf{L} \in \mathcal{L}$ and let $\mathbf{X}_{\mathcal{T}}=\left(\mathcal{L}^{\mathrm{mp}}(\mathbf{L}, \underline{\mathbf{2}}), E, \mathcal{T}\right)$. The lattice $\mathrm{C}(\mathbf{X})=\mathcal{G}^{\mathrm{mp}}\left(\mathrm{D}^{\mathrm{b}}(\mathbf{L}), \underset{\sim}{\mathbf{2}}\right)$ is a compact completion of $\mathbf{L}$. 
Proof Let $A, B \subseteq L$ such that $\bigwedge\left\{e_{a} \mid a \in A\right\} \leqslant \bigvee\left\{e_{b} \mid b \in B\right\}$ in the completion $\mathrm{C}(\mathbf{X})$. Let $\varphi=\bigwedge\left\{e_{a} \mid a \in A\right\}$ and $\psi=\bigvee\left\{e_{b} \mid b \in B\right\}$. We have

$$
\varphi^{-1}(1)=\bigcap_{a \in A} e_{a}^{-1}(1)=\bigcap_{a \in A} W_{a} \quad \text { and } \quad \psi^{-1}(0)=\bigcap_{b \in B} e_{b}^{-1}(0)=\bigcap_{b \in B} V_{b} .
$$

Now $\varphi \leqslant \psi$ in $\mathrm{C}(\mathbf{X})$ means $\varphi^{-1}(1) \subseteq \psi^{-1}(1) \subseteq X \backslash \psi^{-1}(0)$, and hence

$$
\bigcap_{a \in A} W_{a} \subseteq X \backslash \bigcap_{b \in B} V_{b}=\bigcup_{b \in B}\left(X \backslash V_{b}\right) .
$$

Since the space $\mathbf{X}_{\mathcal{T}}$ is compact, and since each $W_{a}$ is $\mathcal{T}$-closed and each $X \backslash V_{b}$ is $\mathcal{T}$-open, the topological compactness yields the existence of finite subsets $A^{\prime}:=$ $\left\{a_{1}, \ldots, a_{r}\right\} \subseteq A$ and $B^{\prime}:=\left\{b_{1}, \ldots, b_{s}\right\} \subseteq B$ such that

$$
W_{a_{1}} \cap \cdots \cap W_{a_{r}} \subseteq\left(X \backslash V_{b_{1}}\right) \cup \cdots \cup\left(X \backslash V_{b_{s}}\right) .
$$

We then let $a^{\prime}:=a_{1} \wedge \cdots \wedge a_{r}$ and $b^{\prime}:=b_{1} \vee \cdots \vee b_{s}$ and observe that

$$
e_{a^{\prime}}^{-1}(1)=W_{a^{\prime}}=\bigcap_{a \in A^{\prime}} W_{a} \subseteq \bigcup_{b \in B^{\prime}}\left(X \backslash V_{b}\right)=X \backslash V_{b^{\prime}}=X \backslash e_{b^{\prime}}^{-1}(0) .
$$

By Lemma 3.7(ii) we get $e_{a^{\prime}}^{-1}(1) \subseteq e_{b^{\prime}}^{-1}(1)$ and so $\bigwedge\left\{e_{a} \mid a \in A^{\prime}\right\} \leqslant \bigvee\left\{e_{b} \mid b \in B^{\prime}\right\}$ as required.

The principal result of this section is now an immediate consequence of Propositions 3.6 and 3.10.

Theorem 3.11 (Canonical extension) Let $\mathbf{L} \in \mathcal{L}$ and let $\mathbf{X}=\left(\mathcal{L}^{\mathrm{mp}}(\mathbf{L}, \underline{\mathbf{2}}), E\right)$. The lattice $\mathrm{C}(\mathbf{X})=\mathcal{G}^{\mathrm{mp}}(\mathbf{X}, \underset{\sim}{\mathbf{2}})$ ordered by

$$
\varphi \leqslant \psi \Longleftrightarrow \varphi^{-1}(1) \subseteq \psi^{-1}(1)
$$

is the canonical extension of $\mathbf{L}$.

We summarise in Fig. 2 what we have achieved. Here

$$
\begin{aligned}
& \mathrm{D}: \mathbf{L} \longmapsto \mathbf{X}_{\mathcal{T}}:=\left(\mathcal{L}^{\mathrm{mp}}(\mathbf{L}, \underline{\mathbf{2}}), E, \mathcal{T}\right), \\
& { }^{\mathrm{b}}: \mathbf{X}_{\mathcal{T}} \longmapsto \mathbf{X}:=\left(\mathcal{L}^{\mathrm{mp}}(\mathbf{L}, \underline{\mathbf{2}}), E\right), \\
& \mathrm{G}: \mathbf{X} \longmapsto \mathrm{C}(\mathbf{X}):=\mathcal{G}^{\mathrm{mp}}(\mathbf{X}, \underset{\sim}{\mathbf{2}}),
\end{aligned}
$$

and $\mathbf{L} \hookrightarrow \mathbf{L}^{\delta}$ via $a \mapsto e_{a}$. The fact that the diagram commutes is the content of Theorem 3.11.

Fig. 2 Factorisation of ${ }^{\delta}$ on objects in $\mathcal{L}$

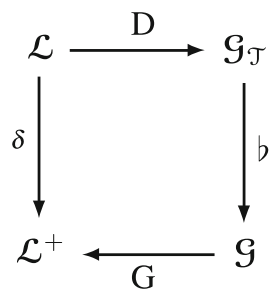


Fig. 3 Seeking to factorise the functor ${ }^{\delta}$ on $\mathcal{L}$

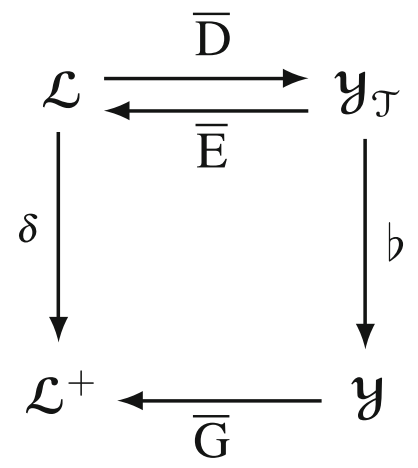

\section{A Categorical Framework for the Canonical Extension}

The canonical extension construction on $\mathcal{L}$ is functorial; see [7, 9]. And, as we observed in Section 1, there is, in the distributive case, a factorisation of the canonical extension functor in terms of functors obtained from the hom-functors setting up Priestley duality. In this section we seek an analogous result for $\mathcal{L}$, insofar as this is possible. Specifically we seek to set up a commutative diagram as shown in Fig. 3, paralleling that shown for $\mathcal{D}$ in Fig. 1.

In Fig. 3 we require

- $\quad \mathcal{L}^{+}$to be the category of complete lattices with complete lattice homomorphisms,

- $\boldsymbol{y}_{\mathcal{T}}$ to be a category of graphs with topology and $\boldsymbol{y}$ the corresponding category of graphs, obtained by forgetting the topology;

- $\overline{\mathrm{D}}$ and $\overline{\mathrm{E}}$ to set up a dual adjunction such that, for all $\mathbf{L} \in \mathcal{L}$, we have $\mathbf{L} \cong \overline{\mathrm{E}} \overline{\mathrm{D}}(\mathbf{L})$ with the isomorphism given by a natural evaluation map,

- $\overline{\mathrm{D}}, \overline{\mathrm{E}}$ and $\overline{\mathrm{G}}$ to be contravariant functors, sending an object $C$ into some appropriately specified set of partial maps into, respectively, $\underline{\mathbf{2}}, \underset{\sim}{\mathbf{2}} \mathcal{T}$ and $\underset{\sim}{\mathbf{2}}$, and

- each of $\overline{\mathrm{D}}, \overline{\mathrm{E}}$ and $\overline{\mathrm{G}}$ to act on morphisms by composition.

Thinking in terms of maps between objects, rather than functors, we have seen (see Fig. 2) that we can obtain a diagram of this kind, based on the Ploščica representation. But regrettably there are inherent obstacles to adding morphisms within Ploščica's framework. For $u \in \mathcal{L}(\mathbf{L}, \mathbf{K})$, the morphism $\mathrm{D}^{\mathrm{b}}(u): \mathrm{D}^{\mathrm{b}}(\mathbf{K}) \rightarrow \mathrm{D}^{\mathrm{b}}(\mathbf{L})$ would need to be given by $\mathrm{D}^{b}(u)(f):=f \circ u$, for all $f \in \mathrm{D}^{b}(\mathbf{K})$, where the domain is determined by the set of elements for which the composition is defined. Certainly, for $f$ a maximal partial homomorphism, $f \circ u$ is a partial homomorphism. But, as the example in Fig. 4 demonstrates, $f \circ u$ need not be maximal.

We have already noted that Ploščica's representation is a recasting of Urquhart's topological representation for bounded lattices. Urquhart was able to set up a dual representation for surjective $\mathcal{L}$-morphisms but not for $\mathcal{L}$-morphisms in general. Therefore our example should come as no surprise. Moreover, it is very easy to see that $f \circ u$ is maximal if $u$ is a surjective morphism and $f$ an MPH.

A solution to the problem of making duality for lattices functorial was proffered by Hartung [11], using topological contexts, but this approach does not fit our needs. Our remedy is quite different. We shall call on the topological representation of 
bounded lattices due to Allwein and Hartonas [1,2]. This is in the same style as that of Urquhart, in that it makes use of disjoint filter-ideal pairs of $\mathbf{L}$ to construct a dual space for $\mathbf{L}$. However such pairs are not required to be maximal, as they are in Urquhart's representation. Allwein and Hartonas thereby overcome the problem encountered by Urquhart and are able to set up a dual equivalence between $\mathcal{L}$ and a specified category of topological structures. This is achieved at the cost of working with a greatly enlarged dual space. A key feature of Priestley duality is its 'logarithmic' property (cf. [3, Section 6.3]). For a finite lattice, moving to a dual space which is bigger than the lattice itself constitutes a major sacrifice, and much of the appeal and power of duality for distributive lattices is lost. This doubtless explains why the work of Allwein and Hartonas has attracted little attention. However the Allwein-Hartonas duality, recast, insofar as this is necessary, in terms of suitable partially defined morphisms, provides exactly the formalism we need to achieve our aim of linking the canonical extension construction to duality in a functorial way, as in Fig 3. We shall now outline what we have to do to carry out our programme.

First of all, we require a Ploščica-style presentation of the Allwein-Hartonas dual spaces. The first task is to recognise which partial homomorphisms from $\mathbf{L}$ into $\underline{\mathbf{2}}$ correspond to disjoint filter-ideal pairs and appropriately to equip the resulting set of partial homomorphisms with the structure of a graph with topology. We may consider the full subcategory $\boldsymbol{y}_{\mathcal{T}}$ of $\boldsymbol{G}_{\mathcal{T}}$ whose objects are the enlarged dual spaces (in our graph formulation, given below) of members of $\mathcal{L}$. We then let $\boldsymbol{y}$ be the image of $\boldsymbol{y}_{\mathcal{T}}$ under ${ }^{b}$. Because of their provenance, we shall refer to the objects of $\boldsymbol{y}$ as L-graphs.

The results of Allwein and Hartonas [1,2] imply that there are contravariant functors $\overline{\mathrm{D}}: \mathcal{L} \rightarrow \boldsymbol{y}_{\mathcal{T}}$ and $\overline{\mathrm{E}}: \boldsymbol{y}_{\mathcal{T}} \rightarrow \mathcal{L}$ which are such that $\mathbf{L} \cong \overline{\mathrm{E}} \overline{\mathrm{D}}(\mathbf{L})$ for every $\mathbf{L} \in \mathcal{L}$; we show that the isomorphism is set up by evaluation maps in the same manner as in Proposition 3.1 (see Propositions 4.3 and 4.4). There is now an obvious candidate for the canonical extension of $\mathbf{L}$ : we forget the topology on $\overline{\mathrm{D}}(\mathbf{L})$ and take the set of all maximal partial morphisms into $\underset{\sim}{\mathbf{2}}$. Rather than verifying directly that we thereby obtain a dense and compact completion we instead set up an orderisomorphism between our new candidate for the canonical extension and the one based on the Ploščica representation. Finally we check out that we really do have a well-defined functor $\overline{\mathrm{G}}$ making the diagram in Fig. 3 commute, and at the same time use our diagram to confirm, in a transparent way, that ${ }^{\delta}$ does lift each $\mathcal{L}$-morphism to a complete lattice homomorphism. We now implement the strategy we have set out.

Let $\mathbf{L} \in \mathcal{L}$. Then the disjoint filter-ideal pairs of $\mathbf{L}$ are obviously in bijective correspondence with those partial homomorphisms $f$ into $\underline{\mathbf{2}}$, for which $\left(f^{-1}(1), f^{-1}(0)\right)$ is a (disjoint) filter-ideal pair of $\mathbf{L}$; let us call them special partial homomorphisms,

Fig. 4 Failure of morphisms to act on MPH's by composition

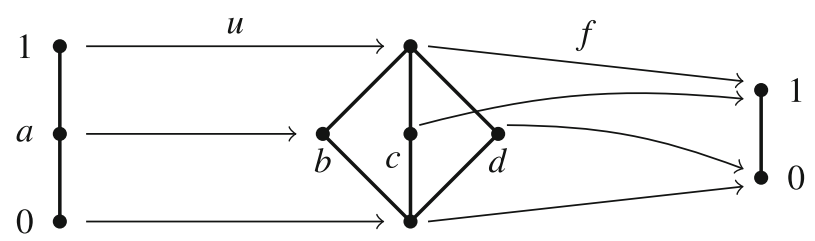

$\mathbf{L}$ $\mathbf{K}$ 
SPH's for short, and denote the set of all such maps by $\mathcal{L}^{\mathrm{sp}}(\mathbf{L}, \underline{\mathbf{2}})$. We note that every maximal partial homomorphism into $\underline{\mathbf{2}}$ is special but that it is easy to find examples of partial homomorphisms into $\underline{\mathbf{2}}$ which fail to be special. The presentation by Allwein and Hartonas now permits a translation into an equivalent formulation in terms of special partial homomorphisms (for the first dual) and continuous partial morphisms (for the second dual). The topology in [2] is defined in the same way as for the representation theorem of Ploščica. That is, let sets of the form

$$
V_{a}=\left\{f \in \mathcal{L}^{\mathrm{sp}}(\mathbf{L}, \underline{\mathbf{2}}) \mid f(a)=0\right\} \quad \text { and } \quad W_{a}=\left\{f \in \mathcal{L}^{\mathrm{sp}}(\mathbf{L}, \underline{\mathbf{2}}) \mid f(a)=1\right\},
$$

form a subbasis for the closed sets of the topology $\mathcal{T}$. We now define the relation $E$ on $\mathcal{L}^{\mathrm{sp}}(\mathbf{L}, \underline{\mathbf{2}})$ by the rule

$$
(f, g) \in E \text { iff } f(x) \leqslant g(x) \text { for every } x \in \operatorname{dom}(f) \cap \operatorname{dom}(g),
$$

that is, we take the obvious extension of $E$, as defined earlier on the subset $\mathcal{L}^{\mathrm{mp}}(\mathbf{L}, \underline{\mathbf{2}})$. We denote the dual space of $\mathbf{L}$ by $\overline{\mathrm{D}}(\mathbf{L})=\left(\mathcal{L}^{\mathrm{sp}}(\mathbf{L}, \underline{\boldsymbol{2}}), E, \mathcal{T}\right)$. The dual space specified in [2], called an enhanced L-space, takes the form of a topological space equipped with two quasi-orders $\left(\leqslant_{1}\right.$ and $\left.\leqslant_{2}\right)$ and a specified subbasis of closed sets.

We shall need the following two lemmas concerning SPH's. The first of these relates the relation $E$ to quasi-orders $\leqslant_{1}$ and $\leqslant_{2}$ (defined on $\mathcal{L}^{\mathrm{sp}}(\mathbf{L}, \underline{\mathbf{2}})$ exactly as on $\left.\mathcal{L}^{\mathrm{mp}}(\mathbf{L}, \underline{\boldsymbol{2}})\right)$. For the case of $\mathcal{L}^{\mathrm{mp}}(\mathbf{L}, \underline{\boldsymbol{2}})$ it appears as Lemma 3.3, which we carried over from [12]; here we show that the result extends to SPH's in general.

Lemma 4.1 Let $\mathbf{L} \in \mathcal{L}$ and let $f, g \in \mathcal{L}^{\mathrm{sp}}(\mathbf{L}, \underline{\mathbf{2}})$. Then

(i) $(f, g) \in E$ if and only if there exists $h \in \mathcal{L}^{\mathrm{sp}}(\mathbf{L}, \underline{\mathbf{2}})$ with $f \leqslant_{1} h$ and $g \leqslant_{2} h$;

(ii) $f \leqslant_{2} g$ if and only if there is no $h \in \mathcal{L}^{\mathrm{sp}}(\mathbf{L}, \underline{\mathbf{2}})$ with $(h, g) \in E$ and $(h, f) \notin E$;

(iii) $f \leqslant_{1} g$ if and only if there is no $h \in \mathcal{L}^{\mathrm{sp}}(\mathbf{L}, \underline{\mathbf{2}})$ with $(g, h) \in E$ and $(f, h) \notin E$.

Proof If $(f, g) \in E$ then $f^{-1}(1) \cap g^{-1}(0)=\emptyset$. Consider $h \in \mathcal{L}^{\mathrm{sp}}(\mathbf{L}, \underline{\mathbf{2}})$ defined by letting $h^{-1}(1)=f^{-1}(1)$ and $h^{-1}(0)=g^{-1}(0)$. Then $f \leqslant_{1} h$ and $g \leqslant_{2} h$. Conversely, if there exists $h \in \mathcal{L}^{\mathrm{sp}}(\mathbf{L}, \underline{\mathbf{2}})$ with $f^{-1}(1) \subseteq h^{-1}(1)$ and $g^{-1}(0) \subseteq h^{-1}(0)$ then clearly $f^{-1}(1) \cap g^{-1}(0)=\emptyset$ and so $(f, g) \in E$. Suppose that $f \leqslant 2 g$ and let $h \in \mathcal{L}^{\mathrm{sp}}(\mathbf{L}, \underline{\mathbf{2}})$. Now suppose that $(h, f) \notin E$. This implies that there exists $a \in L$ such that $h(a)=1$ and $f(a)=0$. Clearly $g(a)=0$ and so $(h, g) \notin E$.

Next, assume that $f \Varangle_{2} g$. This implies that there exists $a \in L$ such that $f(a)=0$ and $g(a) \neq 0$. Consider $h \in \mathcal{L}^{\mathrm{sp}}(\mathbf{L}, \underline{\mathbf{2}})$ defined by $h^{-1}(1)=\uparrow a$ and $h^{-1}(0)=g^{-1}(0)$. Clearly $(h, g) \in E$ and $(h, f) \notin E$, completing the proof. Part (iii) is proved in the same way.

We now derive an extension of Lemmas 3.5 and 3.7 from $\mathrm{D}^{b}(\mathbf{L})$ to $\overline{\mathrm{D}}^{\mathrm{b}}(\mathbf{L})$ :

Lemma 4.2 Let $\mathbf{L} \in \mathcal{L}$. Let $\mathbf{Y}=\left(\mathcal{L}^{\mathrm{sp}}(\mathbf{L}, \underline{\mathbf{2}})\right.$, E) and let $\varphi \in \mathcal{G}^{\mathrm{mp}}(\mathbf{Y}, \underset{\sim}{\mathbf{2}})$ and $f, g \in Y$. Then

(i) if $f \leqslant_{2} g$ and $\varphi(f)=0$, then $\varphi(g)=0$;

(ii) if $f \leqslant 1$ and $\varphi(f)=1$, then $\varphi(g)=1$.

Further, for $a \in L$, we have

(iii) $\varphi^{-1}(0) \cap W_{a}=\emptyset$ implies $\varphi^{-1}(0) \subseteq V_{a}$;

(iv) $\varphi^{-1}(1) \cap V_{a}=\emptyset$ implies $\varphi^{-1}(1) \subseteq W_{a}$. 
Proof Let $f \leqslant_{2} g$ and $\varphi(f)=0$. Then by Lemma 2.1, for all $h \in \varphi^{-1}$ (1) we have $(h, f) \notin E_{Y}$, that is, $h^{-1}(1) \cap f^{-1}(0) \neq \emptyset$. From $f \leqslant 2 g$ we have $f^{-1}(0) \subseteq g^{-1}(0)$, and then clearly for all $h \in \varphi^{-1}(1)$ we have $h^{-1}(1) \cap g^{-1}(0) \neq \emptyset$, whence $(h, g) \notin E_{Y}$. Thus $g \in \varphi^{-1}(0)$, again by Lemma 2.1 .

Now consider part (iii). Let $\varphi^{-1}(0) \cap W_{a}=\emptyset$ and suppose that $f \notin V_{a}$ but $f \in$ $\varphi^{-1}(0)$. Since $f(a) \neq 0$, consider the SPH $g_{f}: \mathbf{L} \rightarrow \underline{\mathbf{2}}$ defined by $g_{f}^{-1}(1)=\uparrow a$ and $g_{f}^{-1}(0)=f^{-1}(0)$. Now $g_{f} \in W_{a}$, but since $f \leqslant 2 g_{f}$ we have by (i) that $g_{f} \in \varphi^{-1}(0)$.

We are now ready to confirm that $\mathbf{L}$ can be recaptured from the graph with topology $\overline{\mathrm{D}}(\mathbf{L})$ in the same manner as, by Ploščica's representation, it is recaptured from $\mathrm{D}(\mathbf{L})$. We first reformulate the representation due to Allwein and Hartonas [1, 2] in terms of graphs with topology in the same way that Ploščica reformulated the $L$ spaces of Urquhart. We want to represent the lattice $\mathbf{L}$ by the maximal partial continuous $E$-preserving maps (MPM's) from $\overline{\mathrm{D}}(\mathbf{L})$ into $\underset{\sim}{\mathbf{2}} \mathcal{T}$.

Let $\mathbf{L} \in \mathcal{L}$, let $\mathbf{Y}_{\mathcal{T}}=\overline{\mathrm{D}}(\mathbf{L})$ and $\mathbf{Y}=\left(\mathcal{L}^{\mathrm{sp}}(\mathbf{L}, \underline{\mathbf{2}}), E\right)$. Let $a \in L$ and define $\bar{e}_{a}: \mathbf{Y}_{\mathcal{T}} \rightarrow$ $\underset{\sim}{\mathbf{2}_{\mathcal{T}}}$ for $f \in \mathcal{L}^{\mathrm{sp}}(\mathbf{L}, \underline{\mathbf{2}})$ by

$$
\bar{e}_{a}(f)= \begin{cases}f(a) & \text { if } a \in \operatorname{dom}(f) \\ - & \text { otherwise }\end{cases}
$$

Proposition 4.3 Let $\mathbf{L} \in \mathcal{L}$ and define $\mathbf{Y}_{\mathcal{T}}=\overline{\mathrm{D}}(\mathbf{L}), \mathbf{Y}=\left(\mathcal{L}^{\mathrm{sp}}(\mathbf{L}, \underline{\mathbf{2}}), E\right)$, and $\bar{e}_{a}$ as above. Then $\bar{e}_{a} \in \mathcal{G}_{\mathcal{T}}^{\mathrm{mp}}\left(\mathbf{Y}_{\mathcal{T}}, \underset{\sim}{\mathbf{2}}\right)$. Furthermore, if $\bar{e}_{a}$ is regarded as a map from $\mathbf{Y}$ to $\underset{\sim}{\mathbf{2}}$, then $\bar{e}_{a} \in \mathcal{G}^{\mathrm{mp}}(\mathbf{Y}, \underset{\sim}{\mathbf{2}})$.

Proof We note that $\bar{e}_{a}^{-1}(1)=W_{a}$ and $\bar{e}_{a}^{-1}(0)=V_{a}$ are closed sets and since the set $\operatorname{dom}\left(\bar{e}_{a}\right)=W_{a} \cup V_{a}$ is closed, too, we have that $\bar{e}_{a}: \operatorname{dom}\left(\bar{e}_{a}\right) \rightarrow \underset{\sim}{\mathbf{2}_{\mathcal{T}}}$ is continuous. Suppose $\bar{e}_{a}(f)=1$ and $\bar{e}_{a}(g)=0$. Then $(f, g) \notin E$ and so $\bar{e}_{a}$ is $E$-preserving. Now assume that $\phi \in \mathcal{G}_{\mathcal{T}}^{\mathrm{mp}}\left(\mathbf{Y}_{\mathcal{T}},{\underset{\sim}{\mathcal{T}}}_{\mathcal{T}}\right)$ and the domain of $\phi: \mathbf{Y}_{\mathcal{T}} \rightarrow \underset{\sim}{\mathbf{2}} \mathcal{T}$ properly extends the domain of $\bar{e}_{a}$. The first case to consider is $f \in \operatorname{dom}(\phi) \backslash \operatorname{dom}\left(\bar{e}_{a}\right)$ such that $\phi(f)=1$. This implies that for all $g \in \bar{e}_{a}^{-1}(0),(f, g) \notin E$. In particular it means that for $h \in \mathcal{L}^{\mathrm{sp}}(\mathbf{L}, \underset{\sim}{\mathbf{2}})$ with $h^{-1}(1)=\{1\}$ and $h^{-1}(0)=\downarrow a$ we have $(f, h) \notin E$. Since $f^{-1}(1)$ is a filter, this can only happen if $f(a)=1$ and so $f \in \operatorname{dom}\left(\bar{e}_{a}\right)$, a contradiction. Similarly one can show that if $\phi(f)=0$ then $f(a)=0$, a contradiction. Thus the domain of $\bar{e}_{a}$ is maximal.

The fact that $\bar{e}_{a} \in \mathcal{G}^{\mathrm{mp}}(\mathbf{Y}, \underset{\sim}{\mathbf{2}})$ follows from Proposition 2.5.

The proof of part (i) of the following result mimics that of [12, Lemma 1.5]. We shall exploit the compactness of the topology $\mathcal{T}$ of $\overline{\mathrm{D}}(\mathbf{L})$ [2, Lemma 3.17].

Proposition 4.4 Let $\mathbf{L} \in \mathcal{L}$ and let $\mathbf{Y}_{\mathcal{T}}=\overline{\mathrm{D}}(\mathbf{L})$.

(i) Every $\varphi \in \mathcal{G}_{\mathcal{T}}^{\mathrm{mp}}\left(\mathbf{Y}_{\mathcal{T}}, \underset{\sim}{\mathbf{2}}\right)$ is of the form $\bar{e}_{a}$ for some $a \in L$.

(ii) $\mathbf{L}$ is order-isomorphic to $\mathcal{G}_{\mathcal{T}}^{\mathrm{mp}}\left(\mathbf{Y}_{\mathcal{T}}, \underset{\sim}{\mathbf{2}}\right)$ via the map $a \mapsto \bar{e}_{a}$.

Proof Since $\varphi$ is $E$-preserving, for any $f \in \varphi^{-1}(1)$ and $g \in \varphi^{-1}(0)$ we get that $(f, g) \notin E$. Thus there must exist $a_{f g} \in L$ such that $f\left(a_{f g}\right)=1$ and $g\left(a_{f g}\right)=0$. We form the set of SPH's $U_{f g}=\left\{h \in \mathcal{L}^{\mathrm{sp}}(\mathbf{L}, \underline{\mathbf{2}}) \mid h\left(a_{f g}\right) \neq 0\right\}$, which is open since 
$U_{f g}=\mathcal{L}^{\mathrm{sp}}(\mathbf{L}, \underline{\mathbf{2}}) \backslash V_{a_{f g}}$. The collection $\left\{U_{f g} \mid f \in \varphi^{-1}(1)\right\}$ is a cover of $\varphi^{-1}(1)$ since $f \in U_{f g}$ for all $f \in \varphi^{-1}(1)$. Now $\varphi^{-1}(1)$ is compact as it is a closed subset of the compact space $\overline{\mathrm{D}}(\mathbf{L})$. Thus there is a finite set $\left\{a_{i} \mid 1 \leqslant i \leqslant n\right\} \subseteq g^{-1}(0)$ such that $\varphi^{-1}(1) \subseteq\left\{\mathcal{L}^{\mathrm{sp}}(\mathbf{L}, \boldsymbol{2}) \backslash V_{a_{i}} \mid 1 \leqslant i \leqslant n\right\}$. We can now set $a_{g}=\bigvee\left\{a_{i} \mid 1 \leqslant i \leqslant n\right\}$. Since $g \in \mathcal{L}^{\mathrm{sp}}(\mathbf{L}, \underline{\mathbf{2}})$, we know that $g^{-1}(0)$ is an ideal and so $g\left(a_{g}\right)=0$. Furthermore, we have that $\varphi^{-1}(1) \subseteq \mathcal{L}^{\mathrm{sp}}(\mathbf{L}, \underline{\mathbf{2}}) \backslash V_{a_{g}}$. From Proposition 2.5 we have that $\varphi \in \mathcal{G}^{\mathrm{mp}}(\mathbf{Y}, \mathbf{2})$ and so using Lemma 4.2(iv) we get $\varphi^{-1}(1) \subseteq W_{a_{g}}$.

Now $\varphi^{-1}(0)$ is covered by open sets of the form $\mathcal{L}^{\mathrm{sp}}(\mathbf{L}, \underline{\boldsymbol{2}}) \backslash W_{a_{g}}$ for $g \in \varphi^{-1}(0)$. Since $\varphi^{-1}(0)$ is also compact, we can take a finite set $\left\{a^{j} \mid 1 \leqslant j \leqslant m\right\}$ so that $\varphi^{-1}(0) \subseteq \bigcup\left\{\mathcal{L}^{\mathrm{sp}}(\mathbf{L}, \underline{\mathbf{2}}) \backslash W_{a^{j}} \mid 1 \leqslant j \leqslant m\right\}$. We also have $\varphi^{-1}(1) \subseteq \bigcap\left\{W_{a^{j}} \mid 1 \leqslant j \leqslant m\right\}$. If we set $a=\bigwedge\left\{a^{j} \mid 1 \leqslant j \leqslant m\right\}$ then $\varphi^{-1}(1) \subseteq W_{a}$ and $\varphi^{-1}(0) \subseteq \mathcal{L}^{\mathrm{sp}}(\mathbf{L}, \underline{\mathbf{2}}) \backslash W_{a}$. Now Lemma 4.2(iii) gives us that $\varphi^{-1}(0) \subseteq V_{a}$ and by the maximality of $\varphi$ we now get $\varphi^{-1}(1)=W_{a}$ and $\varphi^{-1}(0)=V_{a}$ and $\operatorname{so} \varphi=\bar{e}_{a}$.

From Proposition 4.3 and part (i) we have $\mathcal{G}_{\mathcal{T}}^{\mathrm{mp}}\left(\mathbf{Y}_{\mathcal{T}}, \mathbf{2}_{\mathcal{T}}\right)=\left\{\bar{e}_{a} \mid a \in L\right\}$. Now the map $a \mapsto \bar{e}_{a}$ is an order-isomorphism from $\mathbf{L}$ onto $\mathcal{G}_{\mathcal{T}}^{\mathrm{mP}}\left(\mathbf{Y}_{\mathcal{T}}, \mathbf{2}_{\mathcal{T}}\right)$ since $a \leqslant b$ if and only if $\bar{e}_{a}^{-1}(1) \subseteq \bar{e}_{b}^{-1}(1)$. To see this, let $a \leqslant b$ and $f \in \bar{e}_{a}^{-1}(1)$ for $f \in \mathcal{L}^{\mathrm{sp}}(\mathbf{L}, \underline{\mathbf{2}})$. Thus $f(a)=1$ and since $f^{-1}(1)$ is a filter, we obtain $f(b)=1$, whence $f \in \bar{e}_{b}^{-1}(1)$ as required. For the converse, let $a \nless b$ in $\mathbf{L}$. Then $(\uparrow a, \downarrow b)$ is a disjoint filter-ideal pair with an associated special partial homomorphism $f: \mathbf{L} \rightarrow \underline{\mathbf{2}}$ with $f^{-1}(1)=\uparrow a$ and $f^{-1}(0)=\downarrow b$. Then $f \in \bar{e}_{a}^{-1}(1)$ but $f \notin \bar{e}_{b}^{-1}(1)$.

We are finally set up to build a canonical extension of a bounded lattice $\mathbf{L}$, analogous to the one in Theorem 3.11 but now based on the larger dual space $\overline{\mathrm{D}}(\mathbf{L})$. We let $Y=\mathcal{L}^{\mathrm{sp}}(\mathbf{L}, \underline{\mathbf{2}})$ and shall show that the complete lattice of maximal partial $E$-preserving maps from the larger graph $\mathbf{Y}=\left(Y, E_{Y}\right)$ into $\mathbf{2}$ is order-isomorphic to the canonical extension from Theorem 3.11. To accomplish this we recall that $\mathbf{X}=\left(\mathcal{L}^{\mathrm{mp}}(\mathbf{L}, \underline{\mathbf{2}}), E_{X}\right)$ and we define a map $\Psi: \mathcal{G}^{\mathrm{mp}}(\mathbf{X}, \underset{\sim}{\mathbf{2}}) \rightarrow \mathcal{G}^{\mathrm{mp}}(\mathbf{Y}, \underset{\sim}{\mathbf{2}})$ such that for $\varphi \in \mathcal{G}^{\mathrm{mp}}(\mathbf{X}, \underset{\sim}{\mathbf{2}})$ and $f \in Y$,

$$
(\Psi(\varphi))(f)= \begin{cases}1 & \text { if } \forall g \in \varphi^{-1}(0)\left((f, g) \notin E_{Y}\right), \\ 0 & \text { if } \forall h\left(\forall g \in \varphi^{-1}(0)\left((h, g) \notin E_{Y} \Rightarrow(h, f) \notin E_{Y}\right)\right), \\ - & \text { otherwise. }\end{cases}
$$

The next proposition establishes, inter alia, that $\Psi$ is well defined.

Proposition 4.5 Let $\mathbf{X}=\left(\mathcal{L}^{\mathrm{mp}}(\mathbf{L}, \underline{\mathbf{2}}), E_{X}\right)$ and let the map $\Psi$ be defined by $(*)$ above. For every $\varphi \in \mathcal{G}^{\mathrm{mp}}(\mathbf{X}, \underset{\sim}{\mathbf{2}})$,

(i) $\varphi^{-1}(1) \subseteq(\Psi(\varphi))^{-1}(1)$ and $\varphi^{-1}(0) \subseteq(\Psi(\varphi))^{-1}(0)$;

(ii) the map $\Psi(\varphi)$ is a maximal E-preserving map from $\mathbf{Y}=\left(\mathcal{L}^{\mathrm{sp}}(\mathbf{L}, \underline{\mathbf{2}}), E_{Y}\right)$ into $\underset{\mathbf{2}}{\sim}$, and consequently, $\Psi(\varphi) \in \mathcal{G}^{\mathrm{mp}}(\mathbf{Y}, \underset{\sim}{\mathbf{2}})$.

Proof Consider (i). Let $f \in \mathcal{L}^{\mathrm{mp}}(\mathbf{L}, \underline{\mathbf{2}})$ such that $f \in \varphi^{-1}(1)$. By Lemma 2.1(ii), for all $g \in \varphi^{-1}(0)$, we have $(f, g) \notin E_{X}$ and so $(f, g) \notin E_{Y}$. Hence by the definition of $\Psi(\varphi)$, we get $f \in(\Psi(\varphi))^{-1}(1)$. If $f \in \varphi^{-1}(0)$, let us consider $h \in \mathcal{L}^{\mathrm{sp}}(\mathbf{L}, \underline{\mathbf{2}})$ such that if $g \in \varphi^{-1}(0)$, then $(h, g) \notin E_{Y}$. Clearly $(h, f) \notin E_{Y}$, which, by the definition of $\Psi(\varphi)$, gives $f \in(\Psi(\varphi))^{-1}(0)$.

Now consider (ii). First we assume that $(\Psi(\varphi))(h)=1$ and $(\Psi(\varphi))(f)=0$ for some $h, f \in \mathcal{L}^{\mathrm{sp}}(\mathbf{L}, \underline{\mathbf{2}})$. Then from the definition of $(\Psi(\varphi))^{-1}(1)$ we get that $(h, g) \notin E_{Y}$ 
for every $g \in \varphi^{-1}(0)$. Using this, we obtain from the definition of $(\Psi(\varphi))^{-1}(0)$ that $(h, f) \notin E_{Y}$. This shows that $\Psi(\varphi)$ is $E_{Y}$-preserving.

Suppose there exists an $E$-preserving map $\phi: \mathbf{Y} \rightarrow \underset{\sim}{\mathbf{2}}$ with $\operatorname{dom}(\Psi(\varphi)) \subsetneq \operatorname{dom}(\phi)$. Let $f \in \operatorname{dom}(\phi) \backslash \operatorname{dom}(\Psi(\varphi))$ for $f \in \mathcal{L}^{\mathrm{sp}}(\mathbf{L}, \underline{\mathbf{2}})$. First, let us consider the case $\phi(f)=1$. Since $f \notin(\Psi(\varphi))^{-1}(1)$, there exists $g \in \varphi^{-1}(0)$ such that $(f, g) \in E_{Y}$. By part (i) we have that $\varphi^{-1}(0) \subseteq(\Psi(\varphi))^{-1}(0)$, and since $(\Psi(\varphi))^{-1}(0) \subseteq \phi^{-1}(0)$, we get $g \in \phi^{-1}(0)$. This yields that $\phi$ is not $E_{Y}$-preserving, a contradiction. Now let $\phi(f)=0$. Since $f \notin(\Psi(\varphi))^{-1}(0)$, there exists $g \in(\Psi(\varphi))^{-1}(1)$ with $(g, f) \in E_{Y}$. But then $g \in \phi^{-1}(1)$ and so $\phi$ is not $E_{Y}$-preserving, a contradiction. We have shown the maximality of $\Psi(\varphi)$.

The following lemma will be deployed in the proof of Theorem 4.7.

Lemma 4.6 Let $\Psi$ be defined as in (*). For $\eta \in \mathcal{G}^{\mathrm{mp}}(\mathbf{Y}, \underset{\sim}{\mathbf{2}})$, let $\varphi_{\eta}: \mathbf{X} \rightarrow \underset{\sim}{\mathbf{2}}$ be the restriction of $\eta$ to $\mathbf{X}$. Then $\varphi_{\eta} \in \mathcal{G}^{\mathrm{mp}}(\mathbf{X}, \underset{\sim}{\mathbf{2}})$ and $\Psi\left(\varphi_{\eta}\right)=\eta$.

Proof It is easy to see from the definition that $\varphi_{\eta}$ is $E_{X}$-preserving. Suppose there exists $\phi: \mathbf{X} \rightarrow \underset{\sim}{\mathbf{2}}$ such that $\operatorname{dom}\left(\varphi_{\eta}\right) \subsetneq \operatorname{dom}(\phi)$. Consider first the case where $f \in$ $\mathcal{L}^{\mathrm{mp}}(\mathbf{L}, \underline{\mathbf{2}})$ with $\widetilde{f} \in \phi^{-1}(1) \backslash \varphi_{\eta}^{-1}(1)$. This implies that there exists $g \in \eta^{-1}(0)$ such that $(f, g) \in E_{Y}$. Consider $h \in \mathcal{L}^{\mathrm{sp}}(\mathbf{L}, \underline{\mathbf{2}})$ defined by $h^{-1}(1)=f^{-1}(1)$ and $h^{-1}(0)=g^{-1}(0)$. We can extend $h$ to $h_{*} \in \mathcal{L}^{\mathrm{mp}}(\mathbf{L}, \underline{\mathbf{2}})$ and by Lemma $4.2(\mathrm{i})$ we get $h_{*} \in \varphi_{\eta}^{-1}(0)$. But $\varphi_{\eta}^{-1}(0) \subseteq \phi^{-1}(0)$ and $(f, h) \in E_{X}$, contradicting $\phi$ being $E_{X}$-preserving. Using the same method we can show that the existence of $f \in \phi^{-1}(0) \backslash \varphi_{\eta}^{-1}(0)$ implies that $\phi$ cannot be $E_{X}$-preserving. Hence $\varphi_{\eta} \in \mathcal{L}^{\mathrm{mp}}(\mathbf{L}, \underline{\mathbf{2}})$.

By the definition of the lattice order of $\mathrm{C}(\mathbf{Y})=\mathcal{G}^{\mathrm{mp}}(\mathbf{Y}, \underset{\sim}{\mathbf{2}})$ (see Theorem 2.3), it suffices to prove $\left(\Psi\left(\varphi_{\eta}\right)\right)^{-1}(1)=\eta^{-1}(1)$. If $f \in \eta^{-1}(1)$ then for all $g \in \eta^{-1}(0)$, we have $(f, g) \notin E_{Y}$ where $f, g \in \mathcal{L}^{\mathrm{sp}}(\mathbf{L}, \underline{\mathbf{2}})$. In particular this applies to every map $g \in \eta^{-1}(0) \cap \mathcal{L}^{\mathrm{mp}}(\mathbf{L}, \underset{\sim}{\mathbf{2}})=\varphi_{\eta}^{-1}(0)$. Hence $f \in\left(\Psi\left(\varphi_{\eta}\right)\right)^{-1}(1)$ and so $\eta^{-1}(1) \subseteq\left(\Psi\left(\varphi_{\eta}\right)\right)^{-1}(1)$.

If $f \notin \eta^{-1}(1)$, there exists $g \in \eta^{-1}(0)$ such that $(f, g) \in E_{Y}$. Let $h \in \mathcal{L}^{\mathrm{sp}}(\mathbf{L}, \underset{\sim}{\mathbf{2}})$ be defined by letting $h^{-1}(1)=f^{-1}(1)$ and $h^{-1}(0)=g^{-1}(0)$. We know that we can extend $h$ to an $h_{*} \in \mathcal{L}^{\mathrm{mp}}(\mathbf{L}, \underset{\sim}{\mathbf{2}})$. Now $g^{-1}(0) \subseteq h_{*}^{-1}(0)$ and so by Lemma 4.2(i) we have that $h_{*} \in \eta^{-1}(0) \cap \mathcal{L}^{\mathrm{mp}}(\tilde{\mathbf{L}}, \underline{\mathbf{2}})=\varphi_{\eta}^{-1}(0)$. Since $f^{-1}(1) \subseteq h_{*}^{-1}(1)$, we have $\left(f, h_{*}\right) \in E_{Y}$ and so clearly $f \notin\left(\Psi\left(\varphi_{\eta}\right)\right)^{-1}(1)$. Thus $\left(\Psi\left(\varphi_{\eta}\right)\right)^{-1}(1) \subseteq \eta^{-1}(1)$.

The next result shows that the complete lattices $\mathcal{G}^{\mathrm{mp}}(\mathbf{X}, \underset{\sim}{\mathbf{2}})$ and $\mathcal{G}^{\mathrm{mp}}(\mathbf{Y}, \underset{\sim}{\mathbf{2}})$ are order-isomorphic under $\Psi$, and that this map fixes the underlying lattice $\mathbf{L}$.

Theorem 4.7 Let $\mathbf{L} \in \mathcal{L}$. Let $\mathbf{X}=\left(\mathcal{L}^{\mathrm{mp}}(\mathbf{L}, \underline{\mathbf{2}}), E_{X}\right), \mathbf{Y}=\left(\mathcal{L}^{\mathrm{sp}}(\mathbf{L}, \underline{\mathbf{2}}), E_{Y}\right)$ and let $\Psi$ be the map defined by $(*)$. Then $\mathcal{G}^{\mathrm{mp}}(\mathbf{X}, \underset{\sim}{\mathbf{2}})$ is order-isomorphic to $\mathcal{G}^{\mathrm{mp}}(\mathbf{Y}, \underset{\sim}{\mathbf{2}})$ under the map $\Psi$.

Moreover, $\Psi\left(e_{a}\right)=\bar{e}_{a}$ for any $a \in L$, where $e_{a} \in \mathcal{G}^{\mathrm{mp}}(\mathbf{X}, \underset{\sim}{\mathbf{2}})$ and $\bar{e}_{a} \in \mathcal{G}^{\mathrm{mp}}(\mathbf{Y}, \underset{\sim}{\mathbf{2}})$ are the evaluation maps representing the elements of $\mathbf{L}$ inside its completions $\mathcal{G}^{\mathrm{mp}}(\widetilde{\mathbf{X}}, \underset{\sim}{\mathbf{2}})$ and $\mathcal{G}^{\mathrm{mp}}(\mathbf{Y}, \underset{\sim}{\mathbf{2}})$, respectively.

Proof The surjectivity of $\Psi$ has been proved. That $\Psi$ is an order-isomorphism can be seen from the fact that $\varphi \leqslant \psi$ if and only if $\varphi^{-1}(1) \subseteq \psi^{-1}(1)$ (see Theorem 2.3), 
and this occurs if and only if $\psi^{-1}(0) \subseteq \varphi^{-1}(0)$ (by Lemma 2.1), which, in turn, occurs if and only if $(\Psi(\varphi))^{-1}(1) \subseteq(\Psi(\psi))^{-1}(1)$ (by the definition of $\left.\Psi\right)$.

The equality $\Psi\left(e_{a}\right)=\bar{e}_{a}$ follows from Lemma 4.6 using the fact that the restriction of $\bar{e}_{a}$ to $\mathbf{X}$ is $e_{a}$.

Combining the preceding theorem with Theorem 3.11 we obtain the following.

Corollary 4.8 Let $\mathbf{L} \in \mathcal{L}$. Then, with respect to the embedding set up by $a \mapsto \bar{e}_{a}$, the complete lattice $\mathrm{C}(\mathbf{Y})=\mathcal{G}^{\mathrm{mp}}(\mathbf{Y}, \mathbf{2})$ is the canonical extension of $\mathbf{L}$.

We are almost ready to define the functors needed in our categorical approach. First, we make some observations about the relationship between the categories we defined earlier: $\mathcal{G}$, of graphs and $E$-preserving maps, and its topological analogue $\mathcal{G}_{\mathcal{T}}$, of graphs with topology and continuous $E$-preserving maps.

From Lemma 4.1 we note that any $\mathcal{L}$-graph $\mathbf{X}=(X, E)$ is automatically equipped with the quasi-orders $\leqslant_{1}$ and $\leqslant_{2}$, as previously defined. Thus, when considering $\mathbf{X}$ it is not necessary explicitly to state that $\leqslant_{1}$ and $\leqslant_{2}$ are part of the structure of $\mathbf{X}$. We note too that on $\underset{\sim}{\mathbf{2}}$ (which is not an $\mathcal{L}$-graph) we have quasi-orders $\leqslant_{1}$ and $\leqslant_{2}$ defined as follows:

$$
\leqslant 1=E=\leqslant \text { and } \leqslant_{2}=E^{-1}=\geqslant \text {. }
$$

We shall use the symbol $\underset{\sim}{\mathbf{2}}$ to denote both the graph $(\{0,1\}, E)$ and the doublyordered set $(\{0,1\}, \leqslant 1, \leqslant 2)$.

The quasi-order relations $\leqslant_{1}$ and $\leqslant_{2}$ were used by Allwein and Hartonas [2] as well as by Urquhart [14]. Furthermore, Urquhart [14] defines two maps $\ell: \wp(X) \rightarrow$ $\wp(X)$ and $r: \wp(X) \rightarrow \wp(X)$, given by

$$
\ell(A)=\left\{f \in X \mid \forall g \in A\left(f \nless_{1} g\right)\right\} \quad \text { and } r(A)=\left\{f \in X \mid \forall g \in A\left(f \not \nless_{2} g\right)\right\} \text {, }
$$

for $A \subseteq X$.

The maps $\ell$ and $r$ are order-reversing on $(\wp(X), \subseteq)$ and form a Galois connection between the $\leqslant_{2}$-increasing subsets of $X$ and the $\leqslant_{1}$-increasing subsets of $X$. A subset $A \subseteq X$ is defined to be $\ell$-stable if $\ell r(A)=A$ and $r$-stable if $r \ell(A)=A$. The following result is a simple consequence of the theory of Galois connections.

Lemma 4.9 Let $\mathbf{X} \in \mathbf{y}$ and let $A \subseteq X$. Then

(i) $A$ is $\ell$-stable if and only if $A=\ell(B)$ for some $\leqslant_{2}$-increasing $B \subseteq X$;

(ii) $A$ is $r$-stable if and only if $A=r(B)$ for some $\leqslant 1_{1}$-increasing $B \subseteq X$.

Now we define morphisms between $\mathcal{L}$-graphs. It is obvious that we would want any such morphism to preserve the two quasi-orders $\leqslant_{1}$ and $\leqslant_{2}$. We also want morphisms to preserve the way in which these two quasi-orders interact with one another. This we achieve by requiring that inverse images of stable subsets of the codomain be stable sets in the domain of the morphism. We state this more precisely below.

Let $\mathbf{X}$ and $\mathbf{Y}$ be $\mathcal{L}$-graphs and $\alpha: \mathbf{X} \rightarrow \mathbf{Y}$. Then $\alpha$ is an $\mathcal{L}$-graph morphism if

(i) $\alpha$ preserves $\leqslant_{1}$ and $\leqslant_{2}$;

(ii) if $A \subseteq Y$ is an $\ell$-stable set, then $\alpha^{-1}\left(r_{Y}(A)\right)=r_{X}\left(\alpha^{-1}(A)\right)$;

(iii) if $A \subseteq Y$ is an $r$-stable set, then $\alpha^{-1}\left(l_{Y}(A)\right)=l_{X}\left(\alpha^{-1}(A)\right)$. 
We can now give formal definitions of our categories $\boldsymbol{y}_{\mathcal{T}}$ and $\boldsymbol{y}$ : the former consists of $\mathcal{L}$-graphs with continuous $\mathcal{L}$-graph morphisms; the latter is obtained by applying ${ }^{b}$ to $\boldsymbol{y}_{\mathcal{T}}$. Lemma 4.10 serves to tell us that category $\boldsymbol{y}$ (respectively $\boldsymbol{y}_{\mathcal{T}}$ ) is a full subcategory of $\mathcal{G}$ (respectively $\mathcal{G}_{\mathcal{T}}$ ).

Lemma 4.10 Let $\mathbf{X}=\left(X, E_{X}\right)$ and $\mathbf{Y}=\left(Y, E_{Y}\right)$ be objects in $\mathbf{y}$ and consider the map $\alpha: \mathbf{X} \rightarrow \mathbf{Y}$. If $\alpha$ is an $\mathcal{L}$-graph morphism, then $\alpha$ is E-preserving.

Proof Let $f, g \in X$ and suppose that $(f, g) \in E_{X}$. Then by Lemma 4.1(i) there exists $h \in X$ such that $f \leqslant_{1} h$ and $g \leqslant_{2} h$. Since $\alpha$ is $\leqslant_{1}$-preserving, we have that $\alpha(f) \leqslant_{1} \alpha(h)$, and since $\alpha$ is $\leqslant_{2}$-preserving, we have $\alpha(g) \leqslant 2 \alpha(h)$. Thus there exists $y=\alpha(h) \in Y$ with the required properties, and so $(\alpha(f), \alpha(g)) \in E_{Y}$.

Not for the first time we are faced with the need to reconcile two notions which might possibly not coincide. We shall now prove that MPE's from $\mathcal{L}$-graphs into $\underset{\sim}{2}$ are exactly the maximal partial $\mathcal{L}$-graph morphisms into $\underset{\sim}{\mathbf{2}}$.

Proposition 4.11 Let $\mathbf{X}=(X, E)$ be an $\mathcal{L}$-graph, and let $\varphi: \mathbf{X} \rightarrow \underset{\sim}{\mathbf{2}}$ be a partial map. Then the following are equivalent:

(1) $\varphi$ is a maximal partial E-preserving map;

(2) $\varphi^{-1}(1)=\ell\left(\varphi^{-1}(0)\right)$ and $\varphi^{-1}(0)=r\left(\varphi^{-1}(1)\right)$;

(3) $\varphi$ is a maximal partial $\mathcal{L}$-graph morphism.

Proof We start by showing the equivalence of (1) and (2). If $\varphi \in \mathcal{G}^{\mathrm{mp}}(\mathbf{X}, \underset{\sim}{\mathbf{2}})$ and $g \in \varphi^{-1}(0)$ then, by Lemma 2.1, for all $f \in \varphi^{-1}(1)$ we have that $(f, g) \notin E$. Since $g \leqslant_{2} f$ implies that $(f, g) \in E$, we have for all $f \in \varphi^{-1}(1)$ that $g \Varangle_{2} f$ and hence $g \in$ $r\left(\varphi^{-1}(1)\right)$. Thus $\varphi^{-1}(0) \subseteq r\left(\varphi^{-1}(1)\right)$. If we suppose that $g \notin \varphi^{-1}(0)$, then by Lemma 2.1 we have that there exists $f \in \varphi^{-1}(1)$ such that $(f, g) \in E$. Now by Lemma 4.1(i) there exists $h$ such that $f \leqslant_{1} h$ and $g \leqslant_{2} h$ and Lemma 4.2(ii) gives us that $h \in \varphi^{-1}$ (1). Thus $g \notin r\left(\varphi^{-1}(1)\right)$ and hence $r\left(\varphi^{-1}(1)\right)=\varphi^{-1}(0)$. We can similarly show that $\ell\left(\varphi^{-1}(0)\right)=$ $\varphi^{-1}(1)$.

Now assume (2) and let $A:=\varphi^{-1}(1)$. To show that $\varphi$ preserves $E$, let $f, g$ be in the domain of $\varphi$, and suppose $(f, g) \in E$ but $\varphi(f)=1$ and $\varphi(g)=0$. From $\varphi(f)=1$ we get $f \in \ell r(A)=\ell\left(\varphi^{-1}(0)\right)$, and $\varphi(g)=0$ gives $g \in r\left(\varphi^{-1}(1)\right)=r(A)$. Lemma 4.1(i) gives us that there exists $h$ such that $f \leqslant_{1} h$ and $g \leqslant_{2} h$. Now $f \in \operatorname{lr}(A)$ means that for all $q \in r(A)$ we have $f \nless_{1} q$ and hence $h \notin r(A)$. This implies that there exists $k \in A$ such that $h \leqslant_{2} k$. The transitivity of $\leqslant_{2}$ then implies that $g \notin r(A)$, a contradiction. In order to see that $\varphi$ is maximal, suppose that $\operatorname{dom} \varphi \subseteq \operatorname{dom} \psi$ for some $\psi \in \mathcal{G}^{\mathrm{mp}}(\mathbf{X}, \underset{\sim}{\mathbf{2}})$. It is clear that $A \subseteq \psi^{-1}(1)$ and $r(A) \subseteq \psi^{-1}(0)$. If $f \in \psi^{-1}(1)$ and $g \in \psi^{-1}(0)$, then $(f, g) \notin E$ and hence $f \psi_{1} g$ and $g \Varangle_{2} f$. Thus any $f \in \psi^{-1}(1)$ must be in $\ell\left(\psi^{-1}(0)\right)$ and similarly $\psi^{-1}(0) \subseteq r\left(\psi^{-1}(1)\right)$. We then get $\psi^{-1}(1) \subseteq$ $\ell\left(\psi^{-1}(0)\right) \subseteq \ell r(A)=A$ and hence $A=\psi^{-1}(1)$. We also have that $r(A)=\psi^{-1}(0)$ and so $\psi=\varphi$, showing that $\varphi$ is maximal. Hence (1) holds.

Now assume (3). Note that $\{0\}$ is $r$-stable and $\{1\}$ is $\ell$-stable in $\underset{\sim}{\mathbf{2}}$ as $r(\{1\})=\{0\}$ and $\ell(\{0\})=\{1\}$. From (ii) and (iii) in the definition of morphisms of $\mathcal{L}$-graphs we get $\varphi^{-1}(0)=\varphi^{-1}(r(\{1\}))=r\left(\varphi^{-1}(1)\right)$ and $\varphi^{-1}(1)=\varphi^{-1}(\ell(\{0\}))=\ell\left(\varphi^{-1}(0)\right)$.

Finally, assuming (2), we get that for any $f, g \in X$, if $\varphi(f)=1$ and $\varphi(g)=0$, then $f \in \ell\left(\varphi^{-1}(0)\right)$ and $g \in r\left(\varphi^{-1}(1)\right)$. By the definition of $\ell$ and $r$ we see that $f \gtrless_{1} g$ 
and $g \nless_{2} f$. Thus $\varphi$ is $\leqslant_{1}$ - and $\leqslant_{2}$-preserving on its domain. Since the sets $\{0\}$ and $\{1\}$ are the $r$ - and $\ell$-stable subsets of $\underset{\sim}{\mathbf{2}}$, we have that $\varphi$ obeys properties (ii) and (iii) required of an $\mathcal{L}$-graph morphism. We now suppose that there exists a partial $\mathcal{L}$-graph morphism $\psi$ from $\mathbf{X}$ to $\underset{\sim}{\mathbf{2}}$ such that $\operatorname{dom}(\varphi) \subseteq \operatorname{dom}(\psi)$. Suppose there exists $f \in \operatorname{dom}(\psi)$ but $f \notin \operatorname{dom}(\varphi)$, and suppose that $\psi(f)=1$. Since $f \notin \varphi^{-1}(1)=$ $\ell\left(\varphi^{-1}(0)\right)$, there exists $g \in \varphi^{-1}(0) \subseteq \psi^{-1}(0)$ such that $f \leqslant 1 g$. This implies that $\psi$ is not $\leqslant 1$-preserving. Similarly, if we suppose that $\psi(f)=0$ we see that $\psi$ will not be $\leqslant 2$-preserving. Hence $\operatorname{dom}(\varphi)$ is maximal. So we have shown (3).

We are, at last, ready to set up the functors we require. We claim that we can define $\overline{\mathrm{D}}: \mathcal{L} \rightarrow \boldsymbol{y}_{\mathcal{T}}$ and $\overline{\mathrm{G}}: \boldsymbol{y} \rightarrow \mathcal{L}^{+}$as follows:

on objects:

on morphisms:
$\overline{\mathrm{D}}: \mathbf{L} \mapsto\left(\mathcal{L}^{\mathrm{sp}}(\mathbf{L}, \underline{\boldsymbol{2}}), E, \mathcal{T}\right)$,

$\overline{\mathrm{D}}: u \mapsto-\circ u$

and

on objects:

on morphisms:

$$
\begin{aligned}
& \overline{\mathrm{G}}: \mathbf{Y} \mapsto \mathcal{G}^{\mathrm{mp}}(\mathbf{Y}, \mathbf{2}), \\
& \overline{\mathrm{G}}: \alpha \mapsto-\circ \alpha .
\end{aligned}
$$

In the action on morphisms of $\bar{D}$ and $\bar{G}$ the domain of the image map is the set on which the composite map is defined. In legitimising these definitions what is at stake is well-definedness: we must ensure that the images of morphisms under $\overline{\mathrm{D}}$ and $\overline{\mathrm{G}}$ are again morphisms for the categories concerned.

Proposition 4.12 Let $\mathbf{L}, \mathbf{K} \in \mathcal{L}$ and let $u: \mathbf{L} \rightarrow \mathbf{K}$ be a lattice homomorphism. Then the map $\overline{\mathrm{D}}^{\mathrm{b}}(u): \overline{\mathrm{D}}^{\mathrm{b}}(\mathbf{K}) \rightarrow \overline{\mathrm{D}}^{\mathrm{b}}(\mathbf{L})$ is an $\mathcal{L}$-graph morphism.

Proof Let $f, g \in \overline{\mathrm{D}}^{b}(\mathbf{K})$ with $f \leqslant \leqslant_{1} g$ and $a \in\left(\left(\overline{\mathrm{D}}^{b}(u)\right)(f)\right)^{-1}(1)=(f \circ u)^{-1}(1)$. This gives us that $u(a) \in f^{-1}(1)$ and hence $u(a) \in g^{-1}(1)$. Thus $a \in\left(\overline{\mathrm{D}}^{\mathrm{b}}(u)\right)(g)^{-1}(1)$ and so $\overline{\mathrm{D}}^{\mathrm{b}}(u)$ is $\leqslant_{1}$-preserving. Likewise, $\overline{\mathrm{D}}^{\mathrm{b}}(u)$ is $\leqslant_{2}$-preserving.

Now consider $A \subseteq \overline{\mathrm{D}}^{b}(\mathbf{L})$ such that $A$ is $\ell$-stable. We want to show that

$$
\left(\overline{\mathrm{D}}^{\mathrm{b}}(u)\right)^{-1}(r(A))=r\left(\left(\overline{\mathrm{D}}^{\mathrm{b}}(u)\right)^{-1}(A)\right) .
$$

Let $f \in \overline{\mathrm{D}}^{b}(\mathbf{K})$ such that $f \notin r\left(\left(\overline{\mathrm{D}}^{b}(u)\right)^{-1}(A)\right)$. This implies that there exists an SPH $g \in\left(\overline{\mathrm{D}}^{b}(u)\right)^{-1}(A)$ such that $f \leqslant_{2} g$. We note that $g \in\left(\overline{\mathrm{D}}^{b}(u)\right)^{-1}(A)$ if and only if $g \circ u \in A$. Now since $\overline{\mathrm{D}}^{b}(u)$ is $\leqslant_{2}$-preserving, we have that $\left(\overline{\mathrm{D}}^{b}(u)\right)(f)=f \circ u \leqslant_{2} g \circ u$ and hence $f \notin\left(\overline{\mathrm{D}}^{b}(u)\right)^{-1}(r(A))$. Thus we have $\left(\overline{\mathrm{D}}^{b}(u)\right)^{-1}(r(A)) \subseteq r\left(\left(\overline{\mathrm{D}}^{b}(u)\right)^{-1}(A)\right)$.

Now if $f \notin\left(\overline{\mathrm{D}}^{b}(u)\right)^{-1}(r(A))$ then $f \circ u \notin r(A)$ and hence there exists $h \in A$ such that $f \circ u \leqslant{ }_{2} h$. Consider the map $g_{h}: \mathbf{K} \rightarrow \underline{\mathbf{2}}$ defined for $b \in \mathbf{K}$ by

$$
g_{h}(b)= \begin{cases}0 & \text { if } b \in f^{-1}(0), \\ 1 & \text { if } b \in \uparrow\left(u\left[h^{-1}(1)\right]\right) \\ - & \text { otherwise. }\end{cases}
$$

We show that $g_{h} \in \mathcal{L}^{\mathrm{sp}}(\mathbf{L}, \underline{\mathbf{2}})$. If $b \in \uparrow\left(u\left[h^{-1}(1)\right]\right)$ then there exists $a \in h^{-1}(1)$ such that $u(a) \leqslant b$. Since $f \circ u \leqslant 2 h$ we have that $h^{-1}(1) \cap(f \circ u)^{-1}(0)=\varnothing$ and this implies $a \notin(f \circ u)^{-1}(0)$. If $b \in f^{-1}(0)$ then $u(a) \in f^{-1}(0)$, a contradiction. Hence $g_{h}^{-1}(1) \cap g_{h}^{-1}(0)=\varnothing$. We need to show that $g_{h}^{-1}(1)$ is a filter of $\mathbf{K}$, so we consider $b_{1}, b_{2} \in g_{h}^{-1}(1)$. By the definition of $g_{h}$, there must exist $a_{1}, a_{2} \in h^{-1}(1)$ such 
that $u\left(a_{1}\right) \leqslant b_{1}$ and $u\left(a_{2}\right) \leqslant b_{2}$. Now since $h^{-1}(1)$ is a filter of $\mathbf{L}$, we have that $a_{1} \wedge a_{2} \in h^{-1}(1)$ and since $u\left(a_{1} \wedge a_{2}\right)$ is a lower bound for $\left\{b_{1}, b_{2}\right\}$ we have that $u\left(a_{1} \wedge a_{2}\right) \leqslant b_{1} \wedge b_{2}$ and so $b_{1} \wedge b_{2} \in g_{h}^{-1}(1)$. Hence $g_{h} \in \mathcal{L}^{\mathrm{sp}}(\mathbf{K}, \underline{\mathbf{2}})$.

If $a \in h^{-1}(1)$ then $u(a) \in u\left[h^{-1}(1)\right]$ and so $u(a) \in g_{h}^{-1}(1)$. Hence $g_{h}(u(a))=1$ and we have $h \leqslant_{1} g_{h} \circ u$. Now since $h \in A=\ln (A)$ and using the definition of $\ell$ and the transitivity of $\leqslant_{1}$, we have that $g_{h} \circ u \in \ell r(A)=A$. Now clearly $f \leqslant_{2} g_{h}$ and since $g_{h} \in\left(\overline{\mathrm{D}}^{b}(u)\right)^{-1}(A)$ we have $f \notin r\left(\left(\overline{\mathrm{D}}^{b}(u)\right)^{-1}(A)\right)$. Thus $r\left(\left(\overline{\mathrm{D}}^{\mathrm{b}}(u)\right)^{-1}(A)\right) \subseteq$ $\left(\overline{\mathrm{D}}^{b}(u)\right)^{-1}(r(A))$.

In order to show the well-definedness of $\overline{\mathrm{G}}$, we must verify that the image of a morphism under $\overline{\mathrm{G}}$ takes MPE's to MPE's. To address this question we first prove a technical lemma.

Lemma 4.13 Let $\mathbf{X}, \mathbf{Y} \in \boldsymbol{y}$ and let $\alpha: \mathbf{X} \rightarrow \mathbf{Y}$ be such that $\alpha \in \mathbf{y}(\mathbf{X}, \mathbf{Y})$. Further, let $\varphi \in \mathcal{G}^{\mathrm{mp}}(\mathbf{Y}, \underset{\sim}{\mathbf{2}})$ and $f \in X$. Then

(i) if there exists $m \in \varphi^{-1}(0)$ such that $(\alpha(f), m) \in E_{Y}$ then there exists $g_{m} \in X$ such that $\varphi\left(\alpha\left(g_{m}\right)\right)=0$ and $\left(f, g_{m}\right) \in E_{X}$;

(ii) if there exists $n \in \varphi^{-1}(1)$ such that $(n, \alpha(f)) \in E_{Y}$ then there exists $g_{n} \in X$ such that $\varphi\left(\alpha\left(g_{n}\right)\right)=1$ and $\left(g_{n}, f\right) \in E_{X}$.

Proof Considering (i), we have from Lemma 2.1 that $\alpha(f) \notin \varphi^{-1}(1)$. This implies that $f \notin \alpha^{-1}\left(\varphi^{-1}(1)\right)=\alpha^{-1}\left(\ell\left(\varphi^{-1}(0)\right)\right)$. Now since $\alpha^{-1}$ preserves $\ell$-stable sets, we have that $f \notin \ell\left(\alpha^{-1}\left(\varphi^{-1}(0)\right)\right)$. This implies that there exists $g_{m} \in \alpha^{-1}\left(\varphi^{-1}(0)\right)$ such that $f \leqslant_{1} g_{m}$. Now clearly $\alpha\left(g_{m}\right) \in \varphi^{-1}(0)$ and $\left(f, g_{m}\right) \in E_{X}$.

Proposition 4.14 Let $\mathbf{X}, \mathbf{Y} \in \mathbf{y}$ and let $\alpha: \mathbf{X} \rightarrow \mathbf{Y}$ be an $\mathcal{L}$-graph morphism. Then for any $\varphi \in \mathcal{G}^{\mathrm{mp}}(\mathbf{Y}, \underset{\sim}{\mathbf{2}})$ it is the case that $(\overline{\mathrm{G}}(\alpha))(\varphi) \in \mathcal{G}^{\mathrm{mp}}(\mathbf{X}, \underset{\sim}{\mathbf{2}})$.

Proof We have from Lemma 4.10 that $\alpha$ is $E$-preserving, and hence we can apply Lemma 2.4 to conclude that $(\overline{\mathrm{G}}(\alpha))(\varphi)$ is a partial $E$-preserving map from $\mathbf{X}$ to $\underset{\sim}{\mathbf{2}}$. We show that its domain is maximal.

Suppose that there exists an $E$-preserving map $\psi$ extending $(\overline{\mathrm{G}}(\alpha))(\varphi)$ and then suppose $f \notin \operatorname{dom}((\overline{\mathrm{G}}(\alpha))(\varphi))$ for some $f \in X$. We want to show that $f \notin \operatorname{dom}(\psi)$. Since $f \notin \operatorname{dom}(\varphi \circ \alpha)$ we know that $(\varphi \circ \alpha)(f) \neq 1$ and hence by Lemma 2.1 there must exist $m_{0} \in \varphi^{-1}(0)$ such that $\left(\alpha(f), m_{0}\right) \in E_{Y}$. Similarly, as $f \notin(\varphi \circ \alpha)^{-1}(0)$ we know that $\varphi(\alpha(f)) \neq 0$ and hence by Lemma 2.1 there exists $m_{1} \in \varphi^{-1}(1)$ such that $\left(m_{1}, \alpha(f)\right) \in E_{Y}$.

Now from Lemma 4.13 we get $g_{m_{0}}, g_{m_{1}} \in \mathbf{X}$ such that $\varphi\left(\alpha\left(g_{m_{1}}\right)\right)=1$ with $\left(g_{m_{1}}, f\right) \in E_{X}$ and $\varphi\left(\alpha\left(g_{m_{0}}\right)\right)=0$ with $\left(f, g_{m_{0}}\right) \in E_{X}$. From this we see that $g_{m_{1}} \in$ $((\overline{\mathrm{G}}(\alpha))(\varphi))^{-1}(1)$ whence $\psi\left(g_{m_{1}}\right)=1$ and $g_{m_{0}} \in((\overline{\mathrm{G}}(\alpha))(\varphi))^{-1}(0)$ whence $\psi\left(g_{m_{0}}\right)=0$. If we now suppose, for contradiction, that $f \in \operatorname{dom}(\psi)$, then from $\left(g_{m_{1}}, f\right) \in E_{X}$ we obtain $1=\psi\left(g_{m_{1}}\right) \leqslant \psi(f)$ and from $\left(f, g_{m_{0}}\right) \in E_{X}$ we obtain $\psi(f) \leqslant \psi\left(g_{m_{0}}\right)=0$, which is impossible.

The next result confirms that the codomain of $\overline{\mathrm{G}}$ really is $\mathcal{L}^{+}$, as our commuting diagram demands. The theorem which follows shows that under ${ }^{\delta}$ an $\mathcal{L}$-morphism lifts to an $\mathcal{L}^{+}$-morphism. We emphasise that the proof of the first result is a completely routine definition chase. By contrast, lattice homomorphisms were regarded 
by Gehrke and Harding [7] as instances of additional operations which are join- and meet-preserving, and their liftings treated using the machinery developed to handle extensions of maps in general.

Proposition 4.15 Let $\mathbf{X}, \mathbf{Y} \in \boldsymbol{y}$ and $\alpha \in \boldsymbol{y}(\mathbf{X}, \mathbf{Y})$. Then $\overline{\mathrm{G}}(\alpha): \mathcal{G}^{\mathrm{mp}}(\mathbf{Y}, \underset{2}{\mathbf{2}}) \rightarrow$ $\mathcal{G}^{\mathrm{mp}}(\mathbf{X}, \underset{\sim}{\mathbf{2}})$ is a complete lattice homomorphism. That is, $\overline{\mathrm{G}}(\alpha) \in \mathcal{L}^{+}(\overline{\mathrm{G}}(\mathbf{Y}), \overline{\mathrm{G}}(\mathbf{X}))$.

Proof We prove that $\overline{\mathrm{G}}(\alpha)$ is meet-preserving. The fact that $\overline{\mathrm{G}}(\alpha)$ is join-preserving will follow by a similar argument. Let $f \in X$ and consider the collection of MPE's $\left\{\varphi_{i} \mid i \in I\right\} \subseteq \mathcal{G}^{\mathrm{mp}}(\mathbf{Y}, \underset{\sim}{\mathbf{2}})$. Then

$$
\begin{aligned}
f \in\left(\bigwedge_{i \in I}\left(\overline{\mathrm{G}}(\alpha)\left(\varphi_{i}\right)\right)\right)^{-1}(1) & \Longleftrightarrow f \in \bigcap_{i \in I}\left(\overline{\mathrm{G}}(\alpha)\left(\varphi_{i}\right)\right)^{-1}(1) \\
& \Longleftrightarrow(\forall i \in I)\left(\varphi_{i} \circ \alpha\right)(f)=1 \\
& \Longleftrightarrow(\forall i \in I) \varphi_{i}(\alpha(f))=1 \\
& \Longleftrightarrow \alpha(f) \in \bigcap_{i \in I} \varphi_{i}^{-1}(1) \\
& \Longleftrightarrow\left(\bigwedge_{i \in I} \varphi_{i}\right)(\alpha(f))=1 \\
& \Longleftrightarrow\left(\left(\bigwedge_{i \in I} \varphi_{i}\right) \circ \alpha\right)(f)=1 \\
& \Longleftrightarrow f \in\left(\overline{\mathrm{G}}(\alpha)\left(\bigwedge_{i \in I} \varphi_{i}\right)\right)^{-1}(1) .
\end{aligned}
$$

We can now present the main result of this section.

Theorem 4.16 Let $u \in \mathcal{L}(\mathbf{L}, \mathbf{K})$ be a homomorphism of bounded lattices. Then

$$
\overline{\mathrm{G}} \overline{\mathrm{D}}^{b}(u): \overline{\mathrm{G}} \overline{\mathrm{D}}^{b}(\mathbf{L}) \rightarrow \overline{\mathrm{G}} \overline{\mathrm{D}}^{\mathrm{b}}(\mathbf{K})
$$

given by the composition of functors $\overline{\mathrm{G}} \circ^{b} \circ \overline{\mathrm{D}}$ is a complete homomorphism of the corresponding canonical extensions.

Proof This follows from Propositions 4.12 and 4.15.

In conclusion, we sum up what we have achieved.

Theorem 4.17 Let the categories $\mathcal{L}, \mathcal{L}^{+}, \boldsymbol{y}_{\mathcal{T}}$ and $\boldsymbol{y}$ be as above and construct $\overline{\mathrm{D}}$ and $\overline{\mathrm{G}}$ as indicated. Then

(i) $\overline{\mathrm{D}}: \mathcal{L} \rightarrow \boldsymbol{y}_{\mathcal{T}}$ and $\overline{\mathrm{G}}: \mathbf{y} \rightarrow \mathcal{L}^{+}$are well-defined functors;

(ii) the functor $\overline{\mathrm{D}}$ has a right adjoint $\overline{\mathrm{E}}$ such that the unit of the adjunction is given by evaluation maps which are isomorphisms;

(iii) with the categories and functors as defined above, the diagram in Fig. 3 commutes, so that the canonical extension functor on $\mathcal{L}$ factorises as the composition $\overline{\mathrm{G}} \circ^{\mathrm{b}} \circ \overline{\mathrm{D}}$. 
Acknowledgements The authors express their thanks to the referee as well as to Miroslav Ploščica for their useful comments with respect to the proof of Proposition 3.10.

\section{References}

1. Allwein, G.: The duality of algebraic and Kripke models for linear logic. Thesis, Indiana University, Bloomington, Indiana (1992)

2. Allwein, G., Hartonas, C.: Duality for bounded lattices. Indiana University Logic Group, Preprint Series, IULG-93-25 (1993)

3. Clark, D.M., Davey, B.A.: Natural Dualities for the Working Algebraist. Cambridge University Press, Cambridge (1998)

4. Craig, A.P.K., Haviar, M.: Reconciliation of approaches to the construction of canonical extensions of bounded lattices. Available at http://andrewcraigmaths.wordpress.com/research/

5. Davey, B.A., Haviar, M., Priestley, H.A.: Boolean topological distributive lattices and canonical extensions. Appl. Categ. Struct. 15, 225-241 (2007)

6. Davey, B.A., Haviar M., Priestley, H.A.: Natural dualities in partnership. Appl. Categ. Struct. (2011). doi:10.1007/s10485-011-9253-4

7. Gehrke, M., Harding, J.: Bounded lattice expansions. J. Algebra 238, 345-371 (2001)

8. Gehrke, M., Jónsson, B.: Bounded distributive lattices with operators. Math. Jpn. 40, 207-215 (1994)

9. Gehrke, M., Priestley, H. A.: Canonical extensions and completions of posets and lattices. Rep. Math. Log. 43, 133-152 (2008)

10. Gehrke, M., Vosmaer, J.: A view of canonical extension. In: Logic, Language and Computation, Proceedings of the Eighth International Tbilisi Symposium, TbiLLC, 2009. Lecture Notes in Computer Science, vol. 6618, pp. 77-100 (2011)

11. Hartung, G.: An extended duality for lattices. In: General Algebra and its Applications, Potsdam, 1992. Res. Exp. Math., vol. 20, pp. 126-142. Heldermann (1993)

12. Ploščica, M.: A natural representation of bounded lattices. Tatra Mt. Math. Publ. 5, 75-88 (1995)

13. Priestley, H.A.: Representation of distributive lattices by means of ordered Stone spaces. Bull. Lond. Math. Soc. 2, 186-190 (1970)

14. Urquhart, A.: A topological representation theory for lattices. Algebra Univers. 8, 45-58 (1978) 